\section{Understanding Models of Support for People Facing Multiple Disadvantage: A Literature Review}

Lindsey McCarthy, Sadie Parr, Stephen Green, Kesia Reeve Centre for Regional Economic and Social Research Sheffield Hallam University September 2020

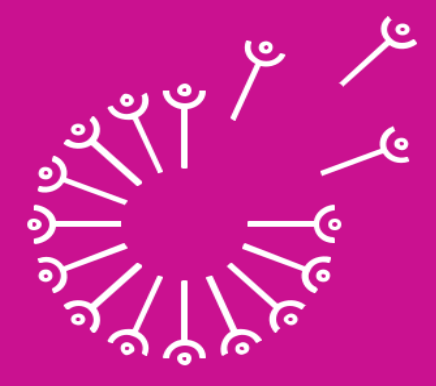

\section{FULFILLING}

\section{LIVES}

LAMBETH

SOUTHWARK LEWISHAM 
Fulfilling Lives Lambeth, Southwark and Lewisham is funded by The National Lottery Community Fund and is part of the National Fulfilling Lives Programme. This is a £112 million investment over 8 years supporting people who are facing multiple disadvantage; the people we work with have a combination and interconnected needs of mental ill-health, are homeless/or at risk of homelessness, substance use and/or offending histories.

Certitude is the lead agency of the programme, delivering the programme in partnership with Thames Reach and strategic partners; South London and Maudsley NHS Trust and the three boroughs of Lambeth, Southwark and Lewisham.

Fulfilling Lives Lambeth Southwark and Lewisham entered a new research and evaluation partnership with New Philanthropy Capital, Groundswell and the Centre for Regional Economic and Social Research in 2020. 


\section{Contents}

1. Introduction

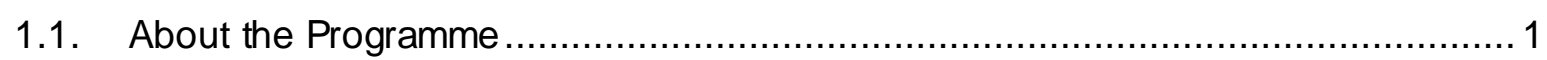

1.2. About the Research and Evaluation Partnership.......................................... 1

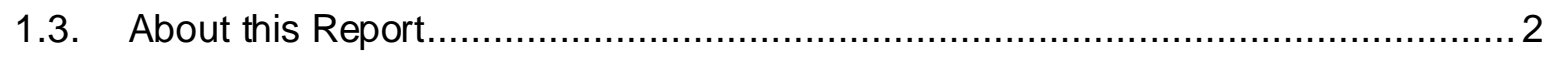

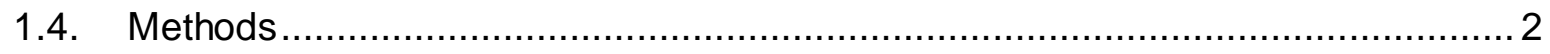

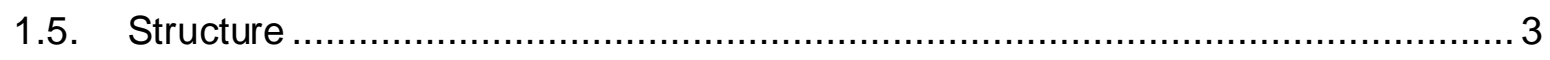

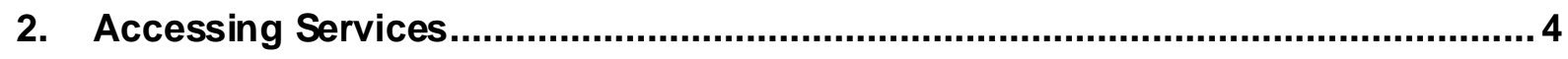

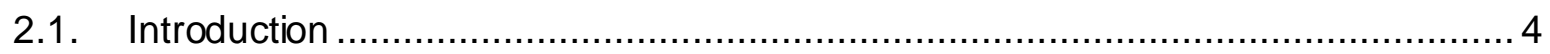

2.2. Multiple disadvantage and service access................................................ 5

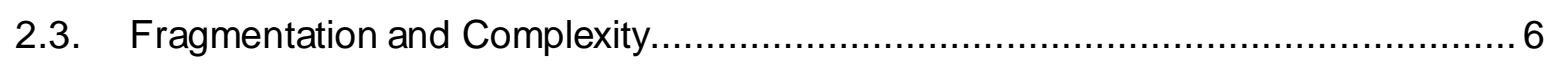

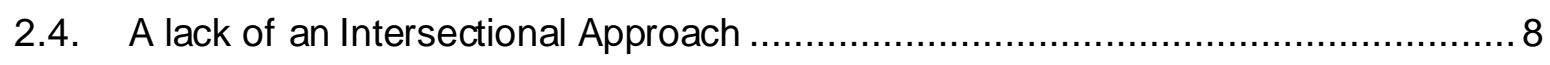

2.5. Service Delivery and Lack of Effective Engagement ........................................ 10

2.6. Funding and Resource Constraints ........................................................... 11

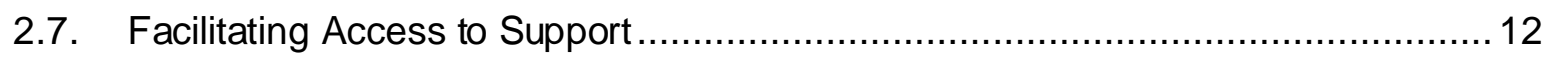

3. The Role of Social and Cultural Norms in Support Journeys ..............................15

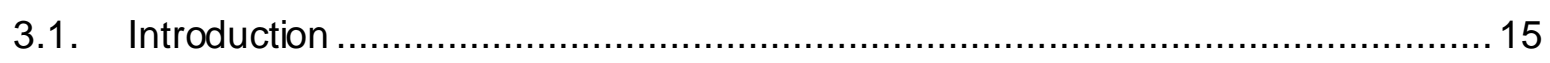

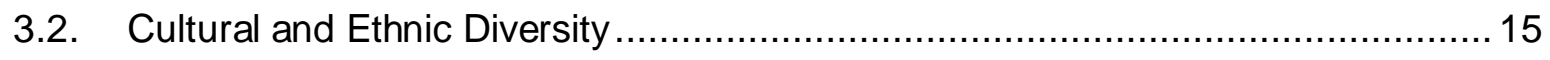

3.3. The role of Gender Norms and Gender Inequality ...................................... 17

4. Trauma-informed Policy and Practice Responses ...........................................21

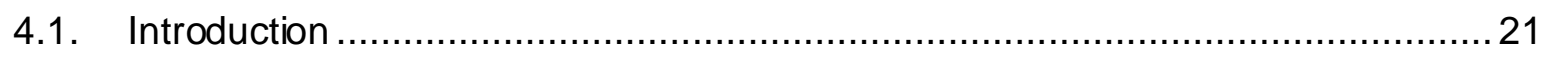

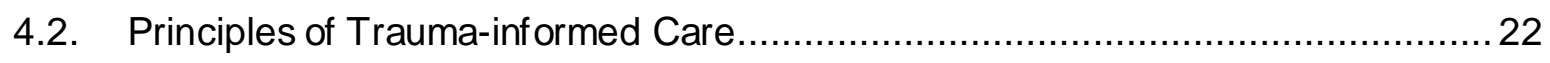

4.3. Trauma-informed Services for People Facing Multiple Disadvantage .................. 23

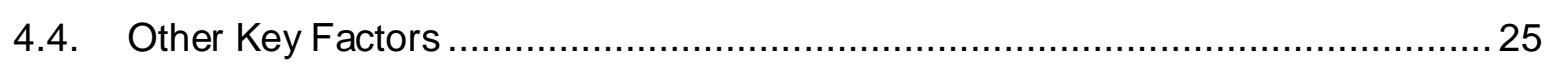

4.5. The Challenges of Implementing a Trauma-informed Approach ...........................29

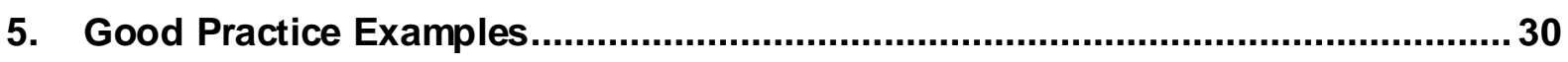

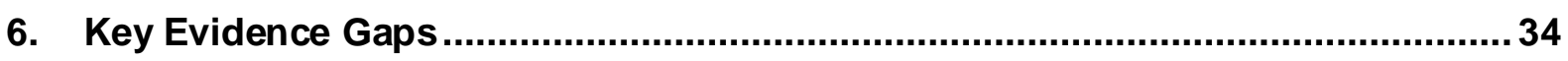

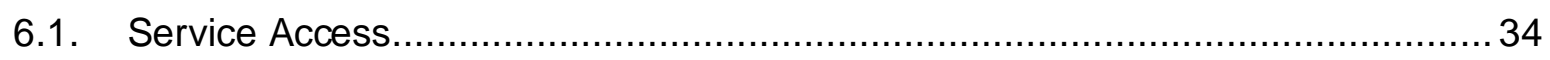

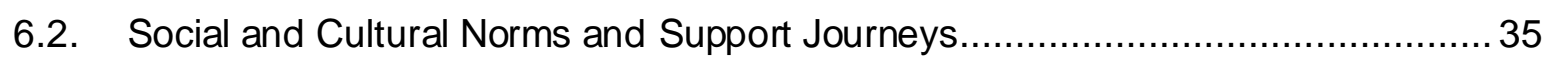

6.3. Trauma-informed Policy and Practice Responses.............................................35

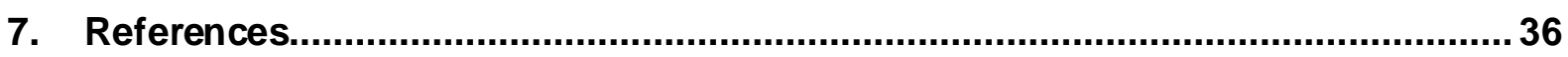




\section{Introduction}

\subsection{About the Programme}

Fulfilling Lives Lambeth, Southwark \& Lewisham (LSL) Programme is funded by The National Lottery Community Fund and is part of the National Fulfilling Lives Programme. Certitude is the lead agency, delivering the programme in partnership with Thames Reach and strategic partners; South London and Maudsley NHS Trust and the three boroughs of Lambeth, Southwark and Lewisham.

Fulfilling Lives LSL works across three main areas:

1. Co-production: it works to develop a culture where people facing multiple disadvantage are at the heart of design and service delivery.

2. Service delivery: it works alongside people and services learning and testing different interventions to make transformative change in order to improve the lives of people facing multiple disadvantage.

3. System change: it provides learning and evidence that will be used to influence on a local and national level, creating sustainable, long-term change for people facing multiple disadvantage. The three system change priorities focus on improving access to support, life transitions and system behaviour i.e. how the system does, or does not, work for people.

\subsection{About the Research and Evaluation Partnership}

NPC, Groundswell and the Centre for Regional Economic and Social Research (CRESR) are working in partnership until the end of 2021 to research systems change and support Fulfilling Lives LSL's interventions. The work aims to build an understanding of effective approaches to improve outcomes for people facing multiple disadvantage. The research aims to:

- Understand the current system: including people's experience of access, transitions and system behaviour, how this is or isn't working, and why.

- Explore how things could change: including the effectiveness of the approaches and models that the programme is testing.

- Start to embed change: including starting to transform local ways of working and strengthening the wider case for system change in order to influence policymakers and commissioners.

NPC, the lead research and evaluation partner, works to improve the impact achieved by social sector organisations. It supports the sector to tackle systemic social issues such as homelessness - through systems change and place-based approaches. 
Groundswell exists to enable people with experience of homelessness take more control of their lives, have a greater influence on services and to play a fuller role in our community. It recruits and trains peer researchers, who have experience of homelessness, to be involved in all stages of the research process. This approach provides genuine and unique grassroots insight into the lives of people facing multiple disadvantage.

CRESR at Sheffield Hallam University focuses on people and places facing multiple disadvantage through its leading academic research. It has expertise in homelessness, vulnerability and public service reform.

\subsection{About this Report}

This literature review brings together broad, multidisciplinary evidence about access to services, transitions within services, and understanding about how the system behaves for people facing multiple disadvantage. The review sought to produce a more detailed understanding about how particular groups within the Fulfilling Lives target population have different experiences due to the diverse effects of social and cultural factors. Related to this, the review also explored extant evidence about culturally and gender-sensitive trauma-informed philosophies underpinning approaches to support. We approach questions about access, transitions and system behaviour through the lens of trauma informed care. The review was guided by the following key questions:

1. What are the systemic factors that prevent (or facilitate) access to and generate productive service encounters for people facing multiple disadvantage?

2. How do pervasive social and cultural norms play a part in the support journeys of particular populations?

3. What are gender and culture-sensitive trauma-informed policy and practice responses to people facing multiple disadvantage?

4. Are there examples of effective trauma-informed care practices in services working with people facing multiple disadvantage?

5. What are the key evidence gaps?

The evidence search was more explicitly focused on literature concerned with gendered and culturally informed understandings of the above questions. Firstly, this is in acknowledgement that these have been lesser explored avenues in the literature overall. Secondly, the search uncovered a wealth of recent literature on gendersensitive approaches to support, and on gender more generally. However, this frequently tended to conflate 'gender' with a focus on women - the current review therefore reflects this focus while still recognising the existence of a larger body of literature on multiple disadvantage and multiple complex needs more generally (i.e. for women and men).

\subsection{Methods}

It is important to acknowledge that the subject matter of this literature review is broadranging and time constraints have meant that we have not been able to cover the considerable body of literature on access, transitions and system behaviour in its entirety. As such this report must be seen as a synopsis which aims to identify and examine some of the key issues rather than provide an in-depth and comprehensive review of all that has been researched and written about the subject. 
Relevant literature was identified through an IDOX Information Services enquiry as well as database searches (Google Scholar, Scopus, and JSTOR) using key search terms ('trauma-informed', 'psychologically informed environments', 'homelessness', 'offending', 'mental health', 'drug and alcohol dependency', 'gender-sensitive' and 'culturally-informed').

Figure 1.1: Search tools
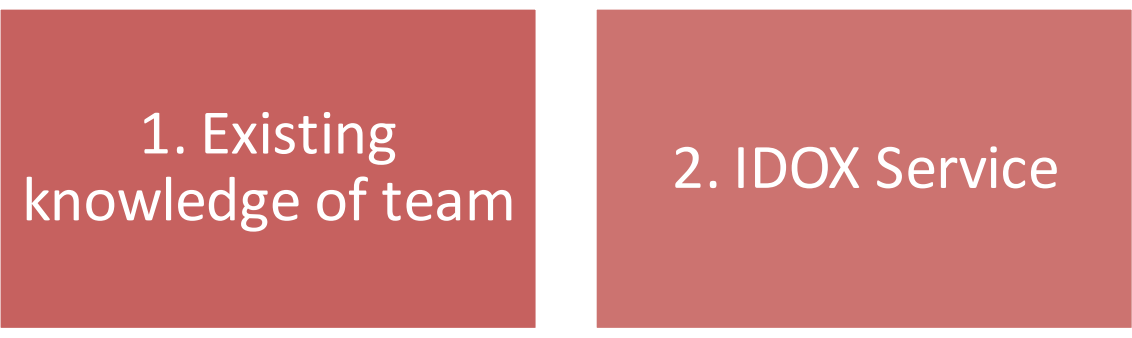

3. Databases and

online libraries

(Google Scholar, JSTOR, Scopus)

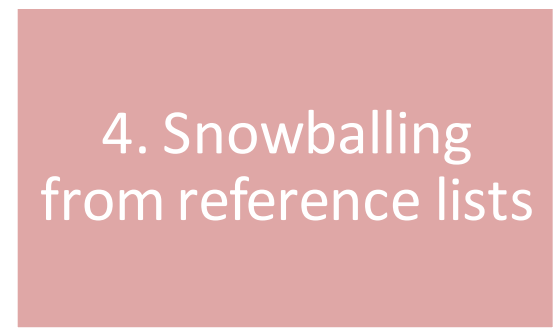

The report focuses primarily on work carried out within the UK that relates to adults and which was published within the last 15 years. Documents searched included academic literature; policy documents; research sponsored by the UK, local and devolved government, charities and service providers; evaluations of policies and programmes; and 'grey literature'. The titles and abstracts (or summaries where abstracts did not exist) of the studies retrieved after the searches were carried out were screened against this inclusion criteria.

\subsection{Structure}

The remainder of this report is divided into five sections.

- $\quad$ Section Two considers systemic barriers to accessing support for people facing multiple disadvantage and factors which help facilitate this access.

- Section Three explores support journeys of particular populations and how these are influenced by pervasive social and cultural norms.

- Section Four discusses how trauma-informed care practices are implemented in services, and how these are gender and culturally-sensitive.

- Section Five presents specific good practice examples of trauma-informed care practices from services working with people facing multiple disadvantage.

- Section Six concludes by outlining the key gaps identified in the literature reviewed. 


\section{Accessing Services}

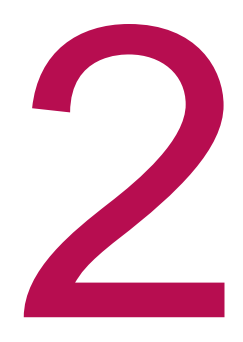

\subsection{Introduction}

This section sets out the existing evidence on systemic barriers that prevent access to support and productive service encounters for groups facing multiple disadvantages in the UK. International literature has also been drawn upon in places where particularly relevant. The section is framed by the following question:

What are the systemic factors that prevent (or facilitate) access to and generate productive service encounters for people facing multiple disadvantage?

The literature search for this section produced an array of sources on multiple needs and exclusions. There is a growing recognition of a significant overlap between groups subject to multiple and extreme forms of disadvantage. However, with exceptions (e.g. Bramley et al., 2015), studies which provide robust evidence on the profile, nature and extent of severe and multiple disadvantage are scarce, and much of it is scattered between reports by charities and service providers focused on their particular client group. There is a clear need for larger-scale and more reliable statistical surveys to provide a more detailed picture of relevant overlaps in order to more robustly make the case that people with multiple needs should be supported by effective, coordinated services. And while there is a relatively large body of evidence on systemic barriers to access for those facing multiple disadvantage, it tends to be fragmented into different groups, some of which receive more focus than others. In a clear break from older studies, which have been criticised for neglecting issues of gender by subsuming their usually small number of female participants into a larger male sample, there is a plethora of recent research focusing solely on women with multiple needs and their access to services. Thus, much of the literature in this section refers to systemic factors that prevent women from accessing services or having productive service encounters. This literature has a tendency to treat women with multiple needs as one group; there is much less evidence on barriers for people from Black, Asian, and minority ethnic (BAME') groups or disabled women facing multiple disadvantage, for instance. Further, there is more work that needs to be done on specific barriers to service access and productive support encounters for particular groups facing multiple disadvantage, most obviously people from BAME groups, LBGT+ people, and people with disabilities.

It is clear from this albeit limited evidence base that there are gaps in understanding in some services around meeting specific needs and accommodating different experiences. The consequences of not doing so are severe. The net effect of this can reinforce an 'inverse care law'; i.e. those with the greatest needs are at greatest risk of getting the least services (Rosengard et al., 2007). While the evidence hints that

\footnotetext{
1 The term 'BAME' is used throughout the report to refer to people from Black, Asian, and minority ethnic groups, except where a specific study applies this term differently, such as 'BAMER' (Black, Asian, Minority Ethnic and Refugee).
} 
flexible and personalised support is the solution, is not robust. There needs to be a deeper understanding of what people actually want, based on studies grounded in the perspectives of people facing multiple disadvantage.

\subsection{Multiple disadvantage and service access}

There is currently a great deal of interest in how best to meet the needs of people who face multiple disadvantage, and/or with multiple needs. When commissioners and service providers talk about people with multiple needs they are usually referring to people who are dealing with, or involved in, three or more of the following: homelessness; offending; substance misuse and/or mental ill-health (Changing Lives, 2018; Lamb et al., 2019a). Having 'complex needs' or 'facing multiple disadvantage' means living with several overlapping issues; often one need creates another or makes other needs worse. Other issues may also feature: experience of domestic abuse; experience of sexual violence and/or child sexual abuse; involvement in sex work; loss of custody of one or more child; and/or physical ill-health. There is evidence emerging of a deepening of these needs as a result of austerity measures, as well as the emergence of needs specifically related to changes to the benefits system (Clinks, 2013).

In addition to the above experiences, an overwhelming percentage of in dividuals with multiple needs have been exposed to additional forms of trauma. The overall picture emerges from several studies with this population of significant levels of repeated trauma often over long periods of time - homelessness itself can also be viewed as a traumatic experience (Hopper et al., 2009). This trauma may result from neglect; psychological abuse, physical abuse, and sexual abuse during childhood; community violence; domestic violence and abuse; combat-related trauma; and disasters (Hopper at al., 2009). Trauma may impact a person's capacity for coping, as well as their sense of safety, ability to self-regulate, their sense of self, perception of control and selfefficacy, and interpersonal relationships. Lived experience of homelessness and trauma has also been shown to lead to reluctance to accept interventions (Magwood et al., 2019). A comprehensive review of the evidence base on trauma-informed care ${ }^{2}$ for people experiencing homelessness suggests that it is futile to attempt to solve the issue of homelessness (and its associated issues of offending, substance use and mental health) without addressing the underlying trauma that is so intricately interwoven with the experience of homelessness by practicing a trauma-informed approach (Hopper et al., 2009). Trauma-informed approaches are explored in greater detail in Chapter 4.

Yet, it is well-acknowledged that mainstream services are often unable to effectively engage those living in such complex situations or address issues of long-term recovery, and people with these needs often go without the help they need. The capacity of emergency hostels to resolve homelessness, for instance, has been in doubt for some time with some suggesting that they promote rather than avert patterns of institutional cycling (Homeless Link, 2018a). Services can have the effect of reinforcing earlier traumatic experiences and causing further harm (Revolving Doors Agency, 2015). A lack of effective support for people with multiple needs can have tragic consequences, sometimes resulting in early death. Since the start of the national Fulfilling Lives programme, they report that 171 people have died (or 5 per cent of those who have engaged with the programme) (Lamb et al., 2019c). Early deaths amongst those experiencing homelessness are also reflected in wider national figures, such as those collected by the Bureau of Investigative Journalism. Findings from the Bureau's 'Dying Homeless' project show that on average 11 homeless people a week

2 Trauma-informed approaches to care have origins in the US, and involve ensuring that organisational practice understands the prevalence and impact of trauma (Holly, 2017). 
died in the UK over the 18 months when data was collected 3 ; more than a quarter of the 796 people were under the age of 40 when they died (McClenaghan, 2019). The Bureau also cites research by University College London, which analysed nearly 4,000 medical records for 600 people who died in English hospitals between 2013 and 2016 and who were homeless when admitted. These records were compared to a similar group of people in terms of age and sex who were in the lowest socio-economic group but who had a home. Those in the homeless group were twice as likely to die of strokes and were more substantially affected by cardiovascular disease as a whole. A third of deaths among the homeless group were from treatable conditions, such as tuberculosis, which can improve with the right care (Aldridge et al., 2019). A lack of accessible and effectiveservices also means an over-reliance on emergency services, which comes at a cost to the public purse. Twenty-seven per cent of people getting help from the national Fulfilling Lives programme attended accident and emergency services (A\&E) at least once during their first three months with the programme (Lamb et al., 2019c). Evidence also shows that people facing multiple disadvantage have repeat and intermittent contact with homelessness, substance misuse, and offending services (Bramley et al., 2015). The costs of multiple needs affect a wide range of agencies and organisations, including HM Courts and Tribunals Service, HM Prison and Probation Service, local police forces, NHS Trusts and Care Commissioning Groups.

The evidence also makes clear that this group rarely receive the treatment they need - they 'fall through the cracks' - whether from specialist mental health services or drug and alcohol services (Dobson, 2019; Lamb et al., 2019c). Many of the mainstream mental health services, such as IAPT (Improving Access to Psychological Therapies), have been deemed inaccessible for people with multiple needs (CFE Research and The University of Sheffield, 2020). Access to secondary mental health care is generally through GP referral. The Primary Medical Care Policy and Guidance Manual from NHS England makes it clear that homeless people should not have to provide ID/proof of address in order to access primary care through a GP. However, research carried out in Stoke on Trent (Wilson \& Astley, 2016) found approximately 75 per cent of GP practices are not following this guidance, suggesting that homeless people are facing limited choices in how and where to seek help with mental ill-health (CFE Research and The University of Sheffield, 2020). In an analysis of responses to Freedom of Information requests sent to English mental health trusts, Lucas et al. (2018) found that under half of the 49 trusts contacted had dedicated arrangements or resources including outreach teams and clinical staff co-located in accommodation and support services for people experiencing homelessness; the remaining trusts indicated that they either had some specific arrangements, such as links between local agencies working with homeless people and existing services, or no dedicated arrangements in place at all. This highlights the gap in the way the needs of the homeless population and those facing multiple disadvantage are addressed by statutory mental health services and adds weight to concerns about homeless people having inequitable access to appropriate care and treatment.

The rest of this chapter goes on to explore the key systemic barriers to service access and productive service encounters for people facing multiple disadvantage as identified in the literature. We found that a lack of joint working, poor coordination and communication impacts on transition between services among people facing multiple disadvantage, which is manifest in different ways.

\subsection{Fragmentation and Complexity}

The prevalence of overlap between issues for people facing multiple disadvantage highlights a need for greater collaboration between public services. The value of

\footnotetext{
${ }^{3}$ Data was collected between October 2017 and March 2019.
} 
working holistically with this population has been emphasised, and it is vital that professionals working in these fields recognise that they are very often working with the same people viewed through different 'lenses' (Bramley et al., 2015).

However, public services have been subject to criticism over their complexity and fragmentation. Services remain siloed, with most provision divided across substance use, mental health, homelessness, or involvement in offending. Driven by bureaucracy, funding and commissioning systems, specialised professionalism and the multiplication of services, Rosengard et al. (2007) suggest that service fragmentation is at the root of poor responses to multiple needs. Broken up into different departments and services, each with their own systems, performance targets and governance arrangements, state services often fail to support people with multiple needs (Duffy \& Hyde, 2014).

Evidence also demonstrates a lack of follow-up support or information (provided to case managers, for example) about people who are disengaged or evicted from a service. In the context of transitions, some evidence points to the need for sensitive and ongoing assessment and review. Rosengard et al. (2007) uncovered numerous examples of problematic assessment, support planning and provision in the context of transitions which delayed people gaining access to the services they needed. This includes leaving criminal justice establishments, hospitals or large hostels. A lack of coordinated provision through joint working, particularly around multiple needs and mental health support is a problem that has been spotlighted for many years. With support being scattered across a range of different agencies, people in need (often experiencing co-occurring mental health issues and substance misuse) find themselves confronted by conflicting eligibility thre sholds (Duffy \& Hyde, 2014). For instance, crisis mental health services may not accept a referral for someone with alcohol or drug dependency (and vice versa) - so that people may feel endlessly passed between services or unable to access services at all (Lamb et al., 2019c). Where people engage with multiple agencies, workers within some services can abdicate responsibility because other service providers are involved with a client. Siloed working also means it is much harder to form a 'critical mass' of advocacy, research or large scale service development with a clear focus on populations at risk (McNeish \& Scott, 2014)

The complexity of the support landscape for women in particular has been highlighted by Holly (2017) and is encapsulated in the diagram in Figure 2.1. Holly (2017) focuses explicitly on women in this study, acknowledging that the starting point for the project was that support services for women experiencing multiple disadvantage are generally scarce, and where they do exist, they can be difficult for women to access. For instance, there is no single local (or national) strategic system which supports or has responsibility for women with multiple needs.

Holly (2017) sought to map what and where specialist support is available for women affected by substance use, mental ill-health, homelessness and offending, and found that all but nine (out of 173) local authority areas across England and Wales are home to at least one type of support for substance use, mental health, homelessness or offending. However, in only nineteen areas in England (none in Wales) do women have access to support for all of these issues (Holly, 2017) suggesting that specialist support for women facing these issues is inconsistently scattered between geographical areas. The existence of multiple funding sources, from local authorities, health commissioners, national funding streams, and voluntary sector grants, also results in a complex network of provision (Holly, 2017). Other studies also highlight the fragmentation of services available to women facing multiple disadvantage (Cameron et al., 2016; Fitzpatrick et al., 2011). Women - in this case, who were homeless reported a lack of communication and co-ordination between different agencies and conflicting advice, reinf orcing the sense that homeless women are 'viewed through a 
succession of separate and uncoordinated professional lenses' (Fitzpatrick et al., 2011: 501).

Figure 2.1: Service complexity (Duffy \& Hyde, 2014)

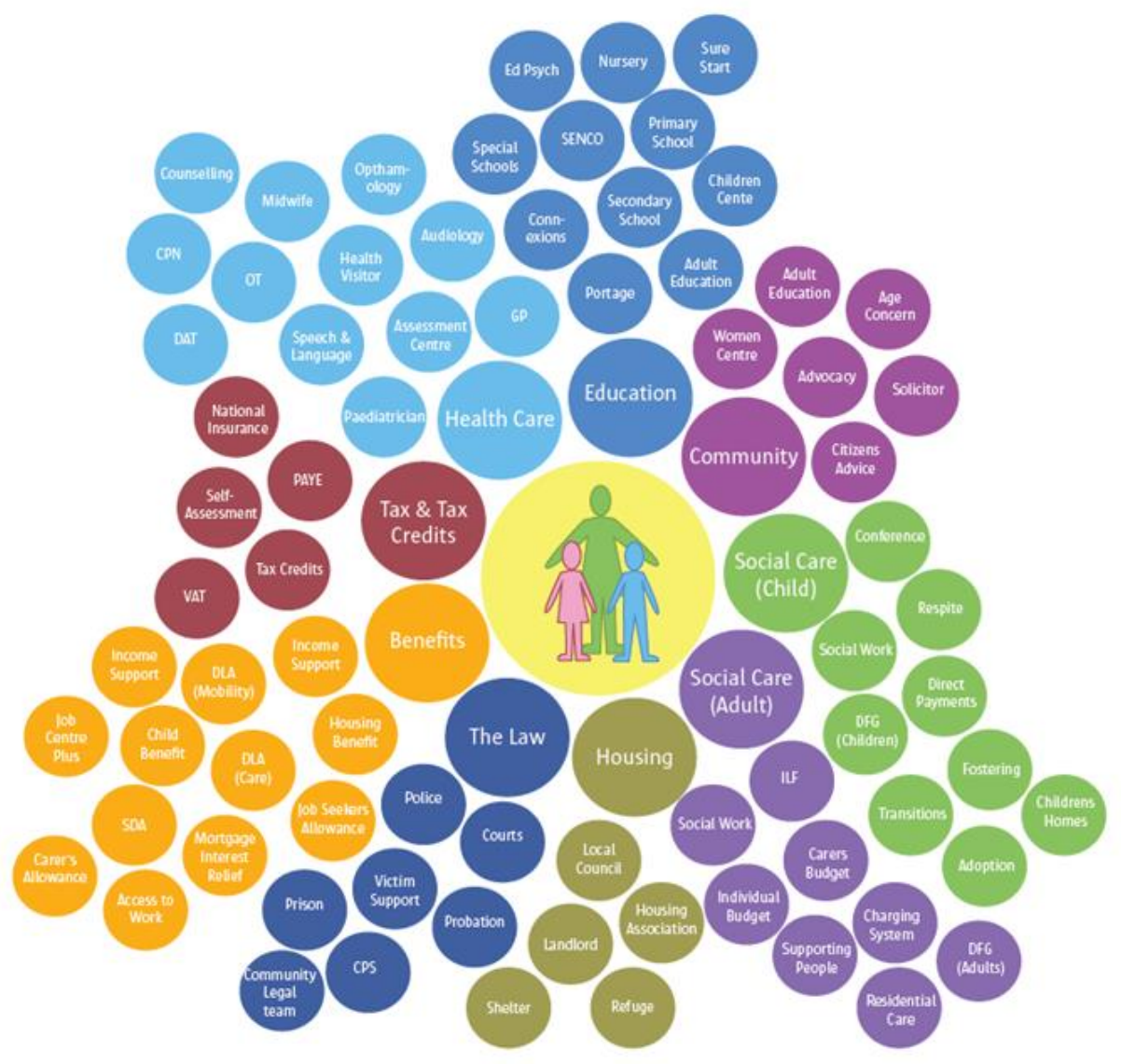

In mapping the type and location of specialist support available for women who experience multiple disadvantage in England and Wales (Holly, 2017), researchers came up against many of the barriers that women face when trying to identify services that may be able to assist them. This included not knowing the right questions to ask to get the answers they need; being over-reliant on the knowledge of the person they ask; not knowing whether they meet the referral criteria; and not knowing how good the service is or whether it still exists. All these barriers are amplified for women at a time of crisis. Overall, the mapping of services currently available to women highlights how the system further disadvantages women by trapping them within a maze - a confusing jumble of paths that often lead nowhere or that give multiple and competing solutions to different points - rather than supporting them to move along and out of the labyrinth of difficulties that characterise their lives. Sharpen's (2018) study revealed a feeling among women with multiple needs that services can often re-traumatise women by the lack of joined-up approaches, causing them to constantly re-tell their stories to multiple practitioners.

\subsection{A lack of an Intersectional Approach}

Policymaking and service design for people who face multiple disadvantage has tended to focus on a set of common issues: homelessness, offending and substance use which has led to a predominant focus on men because they identify as having higher rates of these three issues. In turn, this has led to predominantly gender-neutral service design. A different balance emerges when the definition of multiple disadvantage is changed to incorporate the impact of violence against women and girls, as Sosenko et al. (2020) do in their follow-up to the Hard Edges report (Bramley 
et al., 2015). Their updated def inition of severeand multiple disadvantage includes not only experience of homelessness and substance misuse (as per their original definition) but also violence and abuse and poor mental health in order to cast more light on gendered differences. By using an alternative definition, they found that "a significant number of women [...] face combinations of severe disadvantage at least as serious as those faced by men and on an equivalent scale" (Sosenko et al., 2020: 9 ). Discussed in more detail below, women who have experienced extensive physical or sexual violence and abuse across their life course also face very high rates of issues such as mental ill-health, addiction, homelessness and poverty. Most women in contact with the criminal justice system have also faced domestic or sexual violence. As well as the importance of bringing a critical gendered lens to understandings of multiple disadvantage, is an intersectional understanding. A woman's race/ethnicity, immigration status, sexuality, socio-economic position and experiences living with disability all impact experiences of multiple disadvantage (Ava and Agenda, 2019).

It has been suggested that people facing multiple disadvantage and who also have protected characteristics as defined by the Equalities Act are more likely to face additional barriers to accessing services (Sharpen, 2018). Respondents in Sharpen's (2018) study suggested that disabled women may find it harder to access some services and a lack of suitable ground floor accommodation was reported in all areas studied. They noted that disabled survivors of abuse may also be regarded as vulnerable adults and that contact with local adult safeguarding teams should be made. It was reported that rates of abuse for LGBT + people appear to be higher than the heterosexual population and they are also vulnerable to homo/bi/trans-phobic abuse. LGBT+ people may fear talking to services if it means they must 'come out' to them as well as discussing their other needs.

The evidence from the National Commission on Domestic and Sexual Violence (NCDV) (Ava and Agenda (2019) highlighted a range of ways in which multiply disadvantaged Black, Asian, Minority Ethnic and Refugee (BAMER) women face additional barriers in accessing support. Women described to peer researchers, for example, how their abusers used their position against them: criticising them for not speaking English, not letting them know their rights and telling them they would not be understood. This was borne out when women tried to access services. For example, one woman described how she had contacted a non-BAMER specialist domestic violence service and they had used a generic interpreting service: she ended up not engaging with them because she felt they did not understand her. For migrant women, accessing help was compounded by a lack of knowledge concerning their rights. Bashir et al.'s (2019) research, although with mostly male participants, echoes this evidence in its finding that the belief systems of some BAME communities were littleknown or understood by mainstream service providers.

Qualitative and quantitative research looking into mental health service usage indicates that asylum seekers are five times more likely to have mental health needs than the general population and yet secondary health care data indicates that refugees and asylum seekers are less likely to receive mental health support than the general population (Mental Health Foundation, 2016). In a recent study, people with Asian ethnicity were found to be under-represented among Fulfilling Lives' beneficiaries across the national programme - it is suggested that Asian people with multiple needs may be a 'hidden population' and that more bespoke approaches are needed to engage and support this group (Lamb et al., 2019a).

Language can be a critical barrier to receiving services in minority ethnic communities but also a lack of trust in mainstream 'white' service providers, leading to a reluctance to engage with or act on the advice these services provided (Bas hir et al., 2019). Other communication blocks limit positive outcomes for people with disabilities, including learning disabilities, sensory impairments and dementia. Evidence suggests these 
problems can be overcome with sensitivity and appropriate resources (Rosengard et al., 2007).

\subsection{Service Delivery and Lack of Effective Engagement}

People facing multiple disadvantage may be discouraged fromengaging with a service because their wishes and expectations differ from those of services (Rosengard et al., 2007). A service user reducing their drug or alcohol use (but not stopping entirely) might be deemed a positive move by clients, whilst practitioners might consider it a failure (Williamson et al., 2013). Strict service engagement criteria, then, can sometimes function as an organisational barrier to support, and 'non-engagement' with services may reflect poorly designed, inappropriate services rather than being a consequence of individual behaviour problems or alack of motivation. Aclear example of this can be seen in the 'treatment first' philosophy, underpinning some ('staircasing') models of service provision, which emphasises stability and recovery as prerequisites of movement towards independence or progress (Johnsen \& Teixeira, 2010). Other services operate on a more conditional ' 3 strikes and you are out' basis. In Sharpen's (2018) study, women felt that some services were quick to label them as not engaging when, actually, the service failed to understand their needs or app ropriately engage with them. This left the women in the study feeling 'thrown aside' and invisible.

Many models of delivery exclude people experiencing multiple disadvantage. People with multiple needs may be 'defined out' of the remit of statutory and/or voluntary sector services because they are assessed as being 'too complex' or 'too challenging'. Mental health services often exclude people who are using substances, for instan ce (NCDSV, 2019). Having to remember appointments, attend at fixed times, and wait for long periods do not consider certain needs. Most services only offer support MondayFriday, 9-5, not accounting for times when certain groups might be more vulnerable. Women reported that evenings were a critical point for them, especially when facing multiple issues, and that they felt vulnerable and unsupported during this time (Sharpen, 2018).

Much of the evidence on limited understandings of specific experiences and needs relates to gender and domestic abuse. Although evidence is still patchy in this area, and of ten small-scale, the studies which do exist suggest that typical domestic violence responses do not work for women with multiple needs (NCDSV, 2019; Sharpen, 2018; Sosenko et al., 2020). In generic service encounters, women facing domestic and sexual violence and multiple disadvantage are very rarely asked by professionals about their experiences of abuse (NCDSV, 2019). Each contact with a range of services is often, therefore, a lost opportunity.

Many practitioners interviewed as part of Sharpen's study felt that women facing multiple disadvantage were too complex to be dealt with at Multi Agency Risk Assessment Conferences (MARAC) - a meeting where information is shared on the highest risk domestic abuse cases - where the focus is on leaving an abusive perpetrator. In some cases, women facing multiple disadvantage may still be with the perpetrator, and many women said that domestic violence was not their top priority for support.

A lack of empathy and understanding of women's situations was the most prevalent barrier for women in Changing Lives' (2018) study. The Changing Lives staff interviewed as part of the research revealed that they had encountered several situations where professionals would announce that they or their agency 'wanted nothing more to do with' an individual woman. Respondents characterised these attitudes as 'victim-blaming' and noted a level of judgement about the woman's actions, without commensurate attention paid to the actions of the perpetrator, or any acknowledgement about limited space for action. Fulfilling Lives partnerships across 
the UK also gave examples of people experiencing multiple disadvantage being refused assessments as symptoms of trauma, such as drug-use, behavioural problems or staying in violent or abusive relationships, are assumed to be 'lifestyle choices' (CFE Research and The University of Sheffield, 2020).

Other studies note the ongoing struggle with local decision makers who do not understand the need for gender-specific services. The specific needs of female offenders were still not seen as embedded in local strategies according to representatives from female offender services (Clinks, 2013). A project described it as commissioners being happy to purchase a non-gender specific service that can tick a box to say "Yes, we run a women's morning". Women-only services run by women, as well as some coordinators/navigators, were described as being more person-centred, appreciated the impacts of abuse and trauma and were women's preferred source of support. Yet despite the importance of the women's sector, Sharpen (2018) found a mixed picture regarding its coordination with other services; several had limited knowledge of or involvement in MEAM (Making Every Adult Matter), for instance, a network of charities that support local areas across the country to develop effective, coordinated services that directly improve the lives of people facing multiple disadvantage.

\subsection{Funding and Resource Constraints}

The impact of substantial funding cuts and resulting pressure on existing services should not be underestimated when considering systemic factors that prevent access to productive service encounters for people facing multiple disadvantage. It is of ten the voluntary and community sector that leads the way in piloting new approaches which are more flexible and suitable for this group (Clinks, 2013). Yet, they often work in the cracks between statutory services, only receive a fraction of the funding, and are among the first to face funding cuts (Duffy \& Hyde, 2014). There has been a longterm underinvestment and marginalisation of mental health, in particular, in public policy and service provision (Elliot, 2016; Gilburt,2015;2018), although it is difficult to obtain reliable data on the levels of funding for mental health services in England.

Commissioners of services are increasingly requiring more outcomes for the same investment (Matharoo \& Davis, 2007), yet many services are struggling to adequately meet existing need (especially in a trauma-informed way) with current staffing levels and dedicate a sufficient amount of time to each individual service user (Holly, 2017). The move towards short-term interventions and funding periods was also denounced by professionals interviewed for their experience of developing policy and delivering services to women affected by multiple needs (Holly, 2017). Funding cuts mean that women may see multiple workers over a short period, which has implications for building relationships of trust (Sharpen, 2018). The evidence highlights how problematic it can be to close cases too early, considering the evidence from professionals that the nature of many women's lives means they may need to access services on multiple occasions, often at short notice when a crisis occurs. Disagreements can also arise between providers and service users if options are constrained by resources in the sense that differing preferences reflect the squeeze of limited resources (Rosengard et al, 2007).

With community and hospital-based mental health services under increasing strain, many are unable to access support until they reach crisis point, and the kind of support they do receive in this situation is deemed to be inadequate. When women and girls are detained under the Mental Health Act, for example, there is a lack of traumainformed care, and many risk re-traumatisation as a result of a lack of routine enquiry into patients' experiences of violence and abuse; inappropriate staffing and wards (including male staff delivering restraint, breaches of single-sex accommodation rules, and sexual assaults on mental health wards); and a lack of hospital beds (leading to 
women being held far away from their homes and families, or in inappropriate settings for longer than is necessary). A lack of suitable housing provision also leads to unacceptable outcomes. Research with multiply disadvantaged homeless women indicates that living in a mixed hostel increased their risk of further abuse and substance use (NCDSV, 2018).

The Clinks (2013) review explored how female offender services (from both Voluntary Community and Social Enterprise and statutory sectors) were coping with a changing policy context and funding environment. Eighty-nine per cent of projects felt their service was less secure than or as insecure as it was 12 months ago. One project described themselves as feeling 'run ragged' by austerity measures and how they had impacted on project resources. It was often seen as perplexing that statutory commissioners did not seem to want to protect their investment in a bespoke service for women, which had been developed with their support. They had interpreted this from the apparent lack of desire by the funder to enable the project to participate in negotiations, secure its future and up-skill staff to aid sustainability.

\subsection{Facilitating Access to Support}

There have been various approaches over the last few years to improve outcomes for adults who face multiple disadvantage. Action was set out in the Social Exclusion Task Force report in 2006, Reaching Out: An Action Plan on Social Exclusion (2006). Referring to the "2.5 per cent of every generation who seem caught in a lifetime of disadvantage and harm", the report considered social exclusion as a phenomenon existing throughout an individual's lifetime, and identified action to be taken in respect of the early years of life, childhood and the teenage years, and adulthood. The report concluded that there was a need for public services to re-organise to better identify and meet the needs of adults facing chronic exclusion. A number of programme evaluations have since highlighted a range of lessons around service delivery and effective engagement with this group. According to Duncan \& Corner (2012) there remains more work to be done on how to define and measure 'successful engagement', both in terms of quantifiable data and more subjective outcomes. The national Fulfilling Lives programme was, in part, established in response to these findings.

\section{System navigation}

Where project staff have taken on brokering and advocacy roles, helping people they support to navigate complex support systems, positive outcomes have been observed. This has included more appropriate use of health services by accompanying clients to appointments at the GP surgery or outpatient clinics, as well as increased access to benefits (Cattell et al., 2011). Navigators play an important role in advocating on behalf of people they support, and standing up for their rights when required. This can be through challenging decisions made by statutory services, persevering with a service and advocating if they feel that a refusal or denial by a service is contrary to policy or legislation (CFE Research and The University of Sheffield, 2020). Navigators also have a vital role in supporting people by preparing and accompanying them to appointments and assessments. Peer support and advocacy models, where an individual with a specific experience supports others experiencing similar challenges, have also been shown to have significant potential in supporting people with multiple needs in different fields including homelessness (The Young Foundation, 2016) and healthcare (MacLellan et al., 2015). An independent evaluation of Groundswell's Homeless Health Peer Advocacy programme - which trains Peer Advocates with previous experience of homelessness to engage homeless people, build their trust, knowledge and motivation to keep well and to access health and care services appropriately - found it resulted in a 68 per cent reduction in missed outpatient appointments; a 42 per cent reduction in unplanned care activity; a probable reduction 
in ongoing care costs due to improved health; and potential efficiency savings through better utilisation of health and care services (The Young Foundation, 2016). A systematic review of the impact of peer support models in healthcare concludes that the ability of the Peer Support Worker to actively engage with other marginalised or excluded individuals based on their unique insight into their own experience supports a therapeutic model of care, and calls for the greater availability of peer support models (MacLellan et al., 2015).

\section{Transition points}

Establishing interventions that target specific transition points in the lives of people facing multiple disadvantages, for example from hostel accommodation to a more secure tenancy, leaving an abusive relationship or finding employment, has also been shown to generate positive outcomes. The success of a project in supporting people with transition points may be measured by changes in an individual's accommodation status, employment status and offending behaviour (Cattell et al., 2011). The Social Care Institute for Excellence (SCIE) undertook a Rapid Evidence Assessment of interventions to tackle homelessness across the world. They found that Assertive Community Treatment ${ }^{4}$ and Critical Time Intervention ${ }^{5}$ models were effective services for people experiencing critical transitions in their lives, such as hospital discharges, prison leavers, offenders leaving the criminal justice system, and recent military veterans (Sheikh \& Teeman, 2018). Acomprehensive review on ending homelessness in the UK by Crisis considers evidence on solutions for care leavers at risk of homelessness. While evidence is relatively weak, they reference a framework for improvements in local areas by St Basil's which states that young people leaving care should be given as much information, choice and control as possible; able to make mistakes and never fall out of the framework; helped to succeed; offered flexible support that adapts to meet their needs; and offered supportive and unconditional relationships. They also looked at the evidence base for prevention services for people at immediate risk of homelessness, and found that successful prevention includes a case management approach which provides personalised solutions for households to avoid homelessness; speedy access to financial support (for short-term financial support with rent, security deposits or utility bills, for example); and the provision of expert advice preferably from a 'case manager' who can assist and advocate in accessing help with welfare entitlements, accommodation and other relevant services (Crisis, 2018).

\section{System change}

By establishing effective partnership working, raising awareness of people's needs or at a more strategic level changing commissioning practices, projects can effect changes in the way in which local services are delivered to the group. To improve the delivery of care of people with co-occurring mental health and drug and alcohol use conditions, Public Health England (2017a) recommendations include pathways of care which will enable collaborative delivery of care by multiple agencies; a named care coordinator for every person; joint commissioning across mental health and alcohol/drugs; and a 24/7 response to people experiencing mental health crisis, including intoxicated people. However, it should be noted that there are limits to the ability to effect system change: as Cattell et al. (2011) note, statutory services are bureaucratic, can be slow to change, have their own rules of procurement and need to meet their own performance measures and targets. This might mean they are

\footnotetext{
${ }^{4}$ https://dualdiagnosis.org/co-occurring-disorders-treatment/assertive-community-treatment/

5 CTI is a time-limited evidence based practice that supports people vulnerable to homelessness during periods of transition. CTI has been applied with armed forces veterans, people with mental illness, people leaving prison, and many other groups. It is a housing-led approach providing rapid access to housing. It also features an intensive case manag ement approach to address the particular n eeds of people once they have security of accommodation (Crisis, 2018).
} 
reluctant to engage (Cattell et al., 2011). Several of the Fulfilling Lives partnerships across the UK have created in-house, bespoke mental health services. Pilot projects, such as those run by Opportunity Nottingham and West Yorkshire-Finding Independence, demonstrate that, when designed appropriately, clinical services can engage and effectively support people experiencing multiple disadvantage. Beneficiaries have received vital psychological support to help them manage mental health conditions and past trauma, allowing them to stabilise their behaviours and cope better day-to-day (CFE Research and The University of Sheffield, 2020). 


\section{The Role of Social and Cultural Norms in Support Journeys}

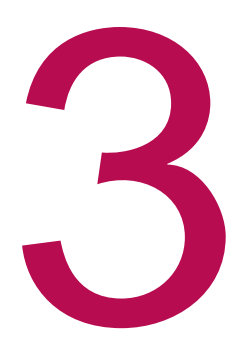

\subsection{Introduction}

This section moves on from the institutional barriers that frustrate access to support, to the social and cultural factors that play a role in the experiences of people who face multiple layers of disadvantage.

While poverty is highlighted in the evidence as a defining feature of the lives of those facing severe and multiple disadvantage, described therein as a "common denominator' (Ava and Agenda, 2019; Johnson and Watt, 2014; Rosengard et al, 2007), social characteristics such as gender, disability, age, race and ethnicity, sexual orientation, and family status also influence the rates and presentation of multiple disadvantage, as well as patterns of access to support and services (Elliott, 2016). This is indicative of the numerous and interconnected social, institutional and personal factors that shape individual support journeys. Among these, pervasive social and cultural norms play a decisive role in increasing the likelihood that some people will experience adverse outcomes, as well as whether and how they access services. This chapter focuses on these social and cultural determinants and addresses the following question:

How do pervasive social and cultural norms play a part in the support journeys of particular populations?

The evidence search identified a disproportionate amount of literature relating to the influence of gender predominantly and, but to a lesser extent, minority ethnic identity. Although we acknowledge that dominant social and cultural norms will be central to the journeys of other marginalised groups such as LGBT+ and disabled people, reflecting the extant evidence available, it is the experiences of women and BAME groups that are considered here. Within the latter section, we talk about the specific needs and experiences of BAME women which have been highlighted (but not considered in sufficient depth) as part of an increasing recognition of the gendered nature of multiple disadvantage. There appears to be less evidence about BAME men's experiences of multiple disadvantage. Both of these groups can be described, to a greater and lesser extent, as 'hidden' within prevailing understandings of multiple disadvantage.

\subsection{Cultural and Ethnic Diversity}

In thinking about how the social and cultural norms associated with an individual's ethnicity impacts on their support journey, it is important to note that the term BAME, which we use here, is one that covers a wide range of people with very diverse experiences and needs. That said, broadly speaking, and in comparison to the majority 
ethnically white population in the UK, BAME groups have different rates of mental health problems, homelessness and substance misuse that reflects their positioning within a wider socio-economic and cultural context (Byrne et al, 2020).

Regarding patterns and prevalence of multiple disadvantage, some key points emerged out of the evidence review. We know for instance that BAME people are over-represented in the mental health system, and that those with African Caribbean heritage are more likely to experience negative or adversarial pathways to care or the 'hard end' of services e.g. compulsory treatment (PHE, 2017). People from BAME groups are also overrepresented in the criminal justice system, representing 25 per cent of the prison population, despite only forming 14 per cent of the overall population (Ministry of Justice, 2017). Although there are marked differences in the rates of homelessness between the various ethnic minority groups, BAME households are significantly more likely to become statutorily homeless than are the majority white population (ODPM, 2005). Poverty is key to understanding this over-representation in homelessness services and statistics - BAME communities are more likely to be living in poverty, in poor standard housing in a deprived area and are more likely to be hidden homeless and living in overcrowded conditions (Homeless Link, 2019). Conversely, BAME groups are often significantly under-represented in mental health services and services treating substance misuse. While there is limited data specifically on BAME groups' experiences of multiple disadvantage, Lamb et al (2019) found that people with Asian ethnicity are under-represented amongst beneficiaries of the national Fulfilling Lives programme, despite some of the projects being in areas with large Asian populations.

The role that cultural norms play in the patterns identified above cannot be overstated, but it is a complex picture. It is also important that by talking about the potential risks presented by social and cultural norms, BAME communities should not be pathologized nor are wider structural causes of harm associated with socio-economic inequality and racism overlooked.

While cultural norms and ties can provide support and belonging, they may also cause harm (EACH, 2009). In Bashir et al's (2019) study of addiction and recovery in Nottingham's BAME community, many of the underlying causes of addiction among BAME people could be found in cultural explanations. This in cluded cultural conflict and the need to 'fit in'. Cultural norms and the social acceptance of alcohol in some cultures were also unlikely to be understood and possibly overlooked. Issues related to shame and stigma likewise played a role in the hidden nature of addiction in many BAME (e.g. Sikh) communities with drug use a particularly taboo issue in the Asian community.

Narratives in Bashir et al. (2019) revealed several reasons behind people's addictions. Trauma, in terms of distressing life events, was prominent, along with ongoing experiences of discrimination, oppression, and racism, which was described as being present throughout people's lives. This reflects wider research that trauma disproportionately affects marginalised populations, including ethnic minorities, and is inseparably bound up with systems of power and oppression (DHSC, 2018; Wilton and Williams, 2019). Social trauma, including poverty, racism and inequality is so prevalent however that it is of ten not recognised as integral to personal adversity such as poor mental health by professionals (Sweeney et al., 2018).

Cultural norms have been found to impact significantly on BAME people's ability to access services and support. The barriers faced by Asian women in particular were highlighted in the evidence review. In relation to domestic violence, Asian women may be more reluctant to speak out for fear of shame, a lack of support in the community or a negative past experience of support, and a range of other cultural pressures. Evidence from a National Commission established to understand the connections 
between women's experiences of domestic and sexual violence and multiple disadvantage found that when BAME women considered trying to seek help from domestic violence services, the fear that they would not be supported or that they may be killed for bringing dishonour on the family, was enough to silence them (Ava and Agenda, 2019). Older research reported a greater cultural tolerance of domestic violence in South Asian communities, underpinned by broader cultural assumptions regarding gender roles and 'family honour' (ODPM, 2005).

In a toolkit developed to disseminate culturally appropriate best practice for professionals, EACH (2009) outline the distinguishing dynamics of domestic abuse experienced by Asian women. They stress that this is not to form stereotypes but to acknowledge some of the additional challenges. These include: multiple abusers (parents, siblings, in laws); more threats of rejection (to divorce, send back to country of origin); rigid gender roles tightly prescribing the role of women; prevalent patriarchal family system; divorced women more severely stigmatised; forced marriages; language and cultural barriers to accessing help. There may also be distinguishing features of physical and sexual violence as well as psychological abuse for Asian women.

Findings from the National Commission (Ava and Agenda, 2019) also pointed to a lack of understanding among practitioners about the links between BAME women's experience of violence and abuse, and other issues, such as substance misuse. Evidence suggests that assumptions can be made about BAME women on the grounds of their faith and culture, for instance that Muslim women will not be using (or misusing) alcohol and drugs, and so substance misuse as a factor can be overlooked.

In comparison to their ethnically white counterparts, some BAME women face additional barriers to accessing mental health support and services. These include issues with recognising and accepting mental health problems, a reluctance to discuss psychological distress and seek help, negative perceptions of and social stigma towards mental health, as well as financial factors (DHSC, 2018).

For many BAME people, it is also important to receive support from tho se who have a shared understanding and experience - of cultural norms, of being from a particular BAME group, of memories and knowledge of a country of origin, of the experience of racism, or prejudice etc. (Matharoo \& Davis, 2007). This also includes a recognition of the importance of faith to some peoples' identity and how some may benefit from having elements of their faith acknowledged and included in proposed support mechanisms and recovery plans (MWNUK, 2019). The literature we identified suggested however that many services do not have a cultural awareness or cultural literacy, and do not have the required skills or capacity to support people experiencing multiple disadvantage who are from a BAME background (Ava and Agenda, 2019). The term 'multiple and complex needs' itself has sometimes been found to be unhelpful and can create a barrier as it is not used nor understood, perceived by some as "eurocentric" and potentially alienating (Bowpitt et al, 2018).

\subsection{The role of Gender Norms and Gender Inequality}

Gender matters in the lives of women and girls at risk of extreme adversity. Gendered social norms and expectations shape risk across the life course of women and this is evidenced in women's different experiences that are associated with multiple disadvantage (e.g. homelessness, mental ill-health, reoffending and addiction).

A great deal of the literature that we reviewed discussed the gendered differences in experiences of mental health problems. This literature emphasises that as well as experiencing mental ill-health at higher rates than men, women experience different conditions from men, experience the same conditions differently, and that much of this 
can be explained by prevailing gender norms (Agenda, 2016). The recent significant increase in mental ill-health among women has led to calls for women's specific needs to be better considered by policy, strategy and services (Wilton and Williams, 2019; Mental Health Foundation, 2017). Young women are a particularly high-risk group, with over a quarter (26 per cent) experiencing a common mental disorder, such as anxiety or depression - almost three times more than young men (9.1 per cent). Complex inter-related factors underlie these trends, but the rise in rates of domestic violence and abuse, together with pressures arising from online culture, social media and pornography are understood as key determinants (Mental Health Foundation, 2017).

There is a strong association between violence and mental ill-health for all women. Research suggests that approximately one in every 20 women in England has experienced extensive physical violence, sexual violence or abuse across their life course (compared to one in every 100 men) and of these, more than half meet the diagnostic criteria for at least one common mental health disorder (Scott \& McManus, 2016). Women's traumatic experiences are significantly more likely to take the form of interpersonal violence and sexual abuse than men's (ibid.) and the more extensive the violence and abuse experienced, the more likely it is that women will also face other adversities too such as substance misuse and homelessness. Economically disadvantaged women are also more likely to experience do mestic violence, and are more likely to experience sexual and physical abuse as both children and adults (McNeish and Scott, 2014).

Gender is pertinent in that it not only increases the risk of violence and abuse, but shapes the ways in which victims respond, and the ways in which others perceive and respond to it. There is evidence that women may have a different first response to threatening situations than men. This difference has been termed 'tend-and-befriend', in contrast to 'fight-or-flight'. 'Tend-and-befriend' refers to an emotion-focused and palliative coping style, with women more likely to seek social support in stressful situations. This reflects evidence that women develop strategies for overcoming and preventing mental health problems that are different to those of men, reflecting wider social and cultural norms (Mental Health Foundation, 2017).

The long-term impact of traumatic experiences also differs by gender. Women are more likely than men to experience psychological harm from trauma, and they are more likely to develop internalising disorders following trauma exposu re. Women have been found to be at higher risk of clinical disorders such as PTSD, anxiety, depression, self-harm, eating disorders and emotionally unstable personality disorder, whereas men have been found to be at greater risk of behavioural difficulties, cognitive misinterpretations of risk, and increased aggression (DHSC, 2018; Wilton and Williams, 2019; Mental Health Foundation, 2017). These gendered responses to trauma are understood within the wider context of men's lives and the role of hegemonic masculinity (Walsh, 2020).

Research suggests that women who face multiple disadvantage are likely to have experienced violent trauma. As McManus et al (2016) found, of the one in 20 women in England who have experienced extensive physical and sexual violence as both a child and an adult, more than half (54 per cent) have a diagnosable mental health condition, 21 per cent have been homeless, 31 per cent have an alcohol problem and 8 per cent are dependent on drugs. Up to a half of women with a dual diagnosis (cooccurrent substance use and mental ill health diagnosis) have experienced sexual abuse (ibid.). However, women facing multiple disadvantage, who have experienced abuse and sexual violence, do not typically present at specialist domestic and sexual violence services and so the links are not always self -evident (Avaand Agenda, 2019). It is important to recognise that definitions of multiple disadvantage have tended to inadvertently exclude women with the focus on the three issues of homelessness, 
offending and substance use, problems that men statistically have higher rates of. If the definition of multiple disadvantage is changed to incorporate the impact of violence against women and girls, a different gender balance emerges (Ava and Agenda, 2019).

For women who experience multiple disadvantage, trauma (such as violent trauma) can be a trigger into offending, substance use and/or homelessness, experiences which in turn cause further trauma which again is differentiated along gendered lines. Bowpitt et al. (2011) used data from a study of multiple exclusion homelessness in Notting ham and London to advance the understanding of the comparable experiences of women and men in becoming, experiencing and addressing homelessness in the context of other multiple needs. While their study was limited to people who made use of services, their findings showed that while there are many similarities in the way men and women experience multiple exclusion homelessness, they do so in the context of a society in which people's opportunities and vulnerabilities are governed by gender relations and associated expectations. Gendered differences were marked in approaches to street survival, with homelessness more likely to be associated with criminal activity among men, but street sex work among women. Women are also less likely than men to appear in rough sleeping counts - and more likely to stay temporarily with friends, family, or strangers, or sleep rough in more hidden (public transport, A\&E waiting rooms) locations - so tend to be underrepresented in some statistics. This can be problematic if service provision is planned on the basis of such statistics (Lamb et al., 2019a; Homeless Link, 2018b).

Despite the evidence, women's experiences of violence and abuse are also rarely recognised as drivers of mental ill-health, offending, addiction and homelessness. Despite the clear relationship for instance between gender based violence and trauma with poor mental health, this link is rarely reflected in the support available to women with mental health problems - service design and delivery frequently fails to take gender into account and trauma informed services are rare (DHSC, 2018). There is evidence that many of those working in a range of services (particularly mental health and criminal justice) are poorly supported to work effectively with women at risk. Their training contains little or nothing about the impact of inequalities or the effects of violence and abuse and does not prepare them for supporting and empowering women and developing services that avoid re-traumatisation and promote recovery (McNeish \& Scott, 2014). While this may have changed since then, this review did not find robust findings to support that.

In research which focused on the different ways that drug and alcohol services and sex work/exiting projects understand the links between women's involvement in prostitution and their substance use (Holly and Lousley, 2014), violence and abuse (by partners and customers) was a common issue for most of the women interviewed. These experiences had a significant and detrimental effect on their mental wellbeing and sense of self; many reported feelings of shame and stigma. Many of the women made the link between feelings of shame, repeated violence and substance use, but felt that drug and alcohol services neglected that link, focusing instead on treating the presenting issue (substance use) rather than the reasons a woman might use (ibid.).

This can lead to situations where services can be inadvertently discriminato ry towards women because they have been designed, whether consciously or unconsciously, around the needs of men. Worse, services can be re-traumatising. For women who have experienced violence and abuse, to be physically restrained within mental health services (including in the face-down position) or put under one to one observation, of ten by male staff, could be profoundly distressing, triggering and humiliating (DHSC, 2018). For those who are homeless, mixed sex service provision risks exposing women to further violence and exploitation and rarely provides the right kind of support (Homeless Link, 2018b). In Mayock et al.'s (2015) research, the material conditions in 
emergency accommodation and the social and emotional stress associated with hostel life acted as push factors towards even riskier housing situations for women. Given the gendered differences in women's experiences of trauma, it is not acceptable for women to simply fit into services which have been designed for men. There is a need to better understand the particular needs of women, how these differ from those of men and (re)design or (re)configure services accordingly (St Mungo's, 2014).

Women's identity as mothers is a key issue that came up repeatedly in the evidence (e.g. DHSC, 2018; Ava and Agenda, 2018; Williamson et al, 2013). Many multiply disadvantaged women have children even if those children are not in their care, temporally or permanently. Many are traumatised by the loss of and separation from children, and struggle to cope with limited or no contact (St Mungo's, 2014). Women who have had their children removed of ten feel as though services abandon them after the removal and that their grief and ongoing sense of loss is not acknowledged (DHSC, 2018; Sharpen, 2018). Conversely, women with children in their care are often reluctant to talk about mental health problems for fear of being considered an unfit mother and their children being take into care. Services' lack of consideration of women's role as mothers also presents practical barriers to women accessing and remaining in mental health and substance misuse services given that few provide childcare. A parallel issue is the inflexibility of some services, by requiring women to attend appointments at certain times of day which is often not achievable. Outreach and one-to-one support available provided by staff around the clock by telephone has been found to be valuable (Holly \& Lousley, 2014).

Closely related to women's identity as a mother, are the twin issues of stigma and shame. Much of what women experience while homeless conflicts with social norms and expectations around being 'good' mothers and maintaining a home. Women feel judged because they do not meet these ideals (Mayock et al, 2015). This perceived failure to live up to these expectations can be a significant barrier both to disclosing problems in assessments and in recovery (St Mungo's, 2014; Rosengard et al, 2007):

We heard again and again how devastating this separation is for women, yet they are often expected to cope with this loss with little or no emotional support. Services are often ill equipped to understand and respond to the further trauma that arises from separation from children and the internalised shame and guilt of 'failing' as a mother. For many womena failure to address this can be a significant blocking factor in recovery from homelessness and wider issues. Providing support in a psychologically informed environment where women have access to emotional support that recognises the significant of separation from children is essential (St Mungo's, 2014:3)

Stigma also plays a role in men's engagement with support services. Gendered concerns around talking about vulnerability and being unemployed can prevent men's participation (Robinson et al, 2015). However, there is striking neglect of and need for further research to engage with the topic of masculinity and the role that hegemonic masculinity plays in the support journeys of men. Nearly all of the material that the review uncovered around 'gender' norms was concerned with the experiences of women. This is because much research and practice has historically been (even if inadvertently so) concerned with the needs of men and women have remained 'hidden' (Reeve, 2018). 


\section{Trauma-informed Policy and Practice Responses}

\subsection{Introduction}

This section explores evidence on trauma-informed care practices - what they are; how they are employed in a gender and culture-sensitive way by services working with people facing multiple disadvantage; and the ongoing chall enges for services of fully implementing them. The section is guided by the following question:

What are gender and culture-sensitive trauma-informed policy and practice responses to people facing multiple disadvantage?

The evidence revealed a range of approaches which seemingly offer hopeful responses to delivering service models for those facing multiple disadvantage. These are presented as enterprising in scope; offering innovative, proactive and preventative models in contrast to traditional conceptions of diagnosis and treatment. The evidence confirms that this needs to be considerable wholesale 'cultural change' across voluntary and statutory services to be effective. In practice, this includes the implementation of trauma-informed care and systems that are rooted in its philosophies (such as Psychologically Informed Environments ${ }^{6}$ and Housing First ${ }^{7}$ ). Recent literature is almost unanimous in its endorsement of trauma-informed care as the 'right' type of intervention. However, others have pointed out how many of its guiding principles have long histories in community and mental health services and the homelessness sector (Dobson, 2019). While there is a bourgeoning evidence base on trauma-informed interventions for those facing multiple disadvantage, it remains under-developed in some respects. Firstly, it tends towards offering descriptive accounts of what constitutes trauma-informed practice rather than critically evaluating or theorising such approaches and their ef fectiveness. Dobson (2019:20) poses the question, for example, 'what do models that embrace complexity delimit and restrict as well as enable for practitioners in their day-to-day thinking about social problems, human experiences and support practice?' Secondly, the evidence is not developed enough to answer whether trauma-informed responses used with people facing multiple disadvantage take into account the variations and complexities of experience between different groups with different support journeys and needs. While the literature on gender-specific responses is now more established, there is less that considers ethnicity and how services might become culture-informed. These gaps represent important future research considerations.

\footnotetext{
${ }^{6}$ Psychologically informed environments (PIE) are services designed and delivered to consider the emotional and psychological needs of the individuals using them and working in them.

${ }^{7}$ See https://hfe.homeless.org.uk/
} 


\subsection{Principles of Trauma-informed Care}

While there appears to be a growing literature on trauma-informed care, it offers little in the way of clarity or consensus on a definition that clearly explains exactly what the concept entails. Instead, what was evident in the literature was a number of common cross-cutting themes that together add to our understanding of trauma-informed care and how organisations can implement it, which are explored below.

Recognising and responding to trauma: trauma-informed care involves ensuring that individual or organisational practice understands the prevalence and impact of trauma; recognises the signs and symptoms of trauma; responds to this knowledge by revising policies, practices and procedures accordingly, and endeavours to ensure that the response from services or systems does not re-traumatise individuals (Harris and Fallot, 2001). Recognising trauma may include facilitating access to counselling, supportive key working and peer support groups to address past and current trauma (DHSC, 2018). A trauma-informed approach understands that people's journeys are defined by both the individual experience and social position, and thereby acknowledges the social roots of trauma and the impact of the context of oppression on clients (DHSC, 2018).

Safe environments: trauma-informed approaches provide, as a minimum, an environment in which trauma is not exacerbated or becomes an impediment to engaging with services. Commonly cited key underlying principles that help to create and sustain such an environment include: trust; collaboration; choice; empowerment; and safety (Vaswani and Paul, 2019).

Staff training, support and supervision: to implement trauma-informed approaches, it is important that services have mechanisms in place to support staff wellbeing. These include robust systems for supervision and debriefing; education about self-care; a culture of trust in which staff are able to say when they are struggling to cope, without fearing that they will be judged or penalised; and sufficient resources to ensure that staff are not expected to shoulder unrealistic workloads. Moreover, there may be fear of moving away fromestablished procedures. It is important that staff work in a traumainformed way because they believe it has value, not simply because policy obliges them to do so. Meaningful training in trauma-informed care is essential here.

Empowering relationships: this is intended to reverse the 'power over' abuses and 'power over' responses to distress that can occur within services such as mental health care, and 'power-over' relationships that disregard the experiences, views and preferences of the individual (Sweeney et al., 2018; 2019). Giving clients a say over how services should be delivered is also mentioned by St Mungo's (2014). Relationships between health and care professionals and people using services are built on respect, compassion and trust (DHSC, 2018).

Strength-based: the fundamental shift in trauma-informed approaches is moving from thinking 'what is wrong with you?' to considering 'what happened to you?' (Sweeney et al, 2018). It will be difficult for a service to work in a trauma-informed way until everyone who is involved in the organisation is willing and able to make the connection between the experiences people have had and the difficulties they face (Holly, 2017). The most successful services for women work from a strengths-based empowerment model, where progress is facilitated by relationships built on faith in the positive possibilities that each woman is capable of achieving (Holly, 2017). This kind of model goes beyond something that is done 'for' women (Ava \& Agenda, 2017).

Equality of access: services promote equality of access to good quality treatment and opportunity for all groups (DHSC, 2018). It is vital that those designing services recognise the diversity of people with multiple needs rather than treating them as a 
uniform group (Lamb et al., 2019a). Services understand the context and conditions of people's lives and are culturally and gender competent (Sweeney et al., 2018).

Wholesale organisational approach: there is agreement in the literature that traumainformed refers to a philosophical stance that requires wholesale change in an organisation's ideology and approach; it cannot be adopted piecemeal but has to apply to all aspects of design and delivery (DHSC, 2018; Holly, 2017). Effective governance and leadership needs to be in place to ensure this change can be initiated and maintained. To support the implementation of cultural change, Covington (2016) recommends that organisations appoint Trauma and Gender Champions. These are individuals tasked with the day-to-day delivery of trauma-informed services and who might serve as role models for all staff on the practical aspects of becoming traumainformed in a manner consistent with the overall culture change goals. The hallmark of trauma-informed care is a "culture of thoughtfulness" in which organisations engage in a continuous process of adaptation and reflection (Wilton and Williams, 2019).

\subsection{Trauma-informed Services for People Facing Multiple Disadvantage}

It is well-acknowledged that people facing multiple disadvantage require tailored responses within policy and services (Elliott, 2016). The evidence base contained some promising approaches to addressing complex trauma, including the development of Psychologically Informed Environments (PIEs) and trauma-informed care practices within services used by people facing multiple disadvantage. However, there is increasing recognition that to be truly trauma-informed, services must recognise the specific needs and experiences of their diverse client group (Lamb et al., 2019a). It is perhaps this principle of 'equality of access' that is less discussed in the literature. This section, therefore, explores evidence on gender and cultureinformed approaches to trauma-informed care.

Sweeney et al. (2018) advocate an understanding of trauma which involves an appreciation of community, social, cultural and historical traumas such as racism, poverty, colonialism, disability, homophobia and sexism and their intersectionality. This means reflecting on racial or cultural biases and creating space for people to explore and def ine their cultural identity; and adopting a 'gender lens' in or der to create safer environments and develop supports that are responsive to the needs and histories of women and men. Each of these lenses is explored in the discussion that follows.

\section{Gender-sensitive approaches}

There is now a wealth of literature on gender-sensitive approach to trauma-informed care. Within this body of work, numerous terms are used to refer to services, policies and practices that recognise and respond to the differences between women and men in their life experiences; and the centrality and importance of gender. These include 'gender-sensitive', 'gender-specific', 'gender-responsive', and 'gender-informed' approaches. Women with lived experience and service providers often had divergent views on women's experiences, how these differ from men, and how this affects their support needs (Sharpen, 2018).

A report by Homeless Link (2018b) notes the distinction between services which take a 'gender-specific approach' and those that provide 'gender-informed support'. The former are services designed and delivered by women and for women, which are usually underpinned by a strong feminist ethos to stand alongside women and support them through a strengths-based approach. The latter are not always women-only and not always underpinned by a feminist approach. Homelessness services supporting women are more likely to fall into this category where they are actively delivering services that recognise women's differing needs from men. The report notes that there 
is a lack of gender-specific services in England. A further study based on Freedom of Information (FOI) requests to 57 mental health foundation trusts in England suggests that the level of consideration given to gender and trauma by NHS Mental Health Trusts is minimal, and in some areas, women are likely to be struggling to get the support they need (Agenda, 2016).

Nevertheless, studies highlight the importance of understanding and embedding elements of a gender-specific approach across services. The importance of employing a gender-specific approach is emphasised by studies which shed light on the consequences of their absence. In one study, a woman describes services as feeling like 'another abuser', taking control of her again. The women felt that a failu re to understand trauma and its impact on current behaviour and presenting needs was a failure to work effectively with women (Sharpen, 2018). Some progress is being made in this respect. The Women's Mental Health Taskforce, in hearing about failings and gaps in current service provision for women, developed a set of gender and traumainformed principles to help commissioners, providers and practitioners consider the specific needs of women with mental illness at a local level (DHSC, 2018: 6). These were developed from statements of what matters to women. A number of organisations and bodies across the health sector (including NHS England, Public Health England, Care Quality Commission, NHS Improvement, Health Education England, and a range of voluntary and community sector organisation) have also made commitments to improve the experiences and treatment of women experiencing mental illness. However, both of these examples identified are in the health sector; it was less clear in the literature what commitments have been made in other sectors (housing, social care, criminal justice services).

A comprehensive review by Ava and Agenda (2017) fleshes out the core components of a gender-sensitive service for women experiencing multiple disadvantage, regardless of sector. Any model of intervention for this group of women must go beyond what is delivered to encompass how it is delivered; the values and approaches underpinning the delivery of a service are just as important as the delivery itself. Echoing this, in research by Homeless Link (2018b), several stakeholders described how their activities and approaches were driven by the overarching aims and ethos which stemmed from the understanding of women's intersectional experiences of inequality.

To achieve a gender-informed approach across mental health services, Agenda (2015) calls for women's needs to be explicitly considered in national mental health policy and strategy; every mental health trust to appoint a clinical lead for women's mental health and a strategy to take into account women's needs; mental health services to implement routine enquiry about women's experiences of violence and abuse which are accompanied by pathways into proper support and care; dedicated women-only services for women with multiple needs to be available in every area; and frontline workers to receive training on women's mental health, trauma, abuse, and to implement trauma-informed practice.

Bailey et al. (2019a) argue that a significant 'culture shift' is required in substance use services in England to embrace an approach that recognises the ubiquity of interpersonal abuse (physical, emotional or sexual violence/abuse in adulthood or childhood) experienced by female users, that is 'non-pathologizing, strengths-based, and centred on 'growth-fostering relationships'. Promising practice shows a commitment to understanding women's multiple disadvantage, including an understanding of intersectional disadvantage, experiences of Violence against Women and Girls (VAWG), and associated trauma (Homeless Link, 2018b). It is estimated that between 40 and 70 per cent of women who use substances have experienced interpersonal abuse, whilst an estimated 30-59 per cent of women receiving substance use treatment have post-traumatic stress disorder (PTSD). These 
interrelated factors, the authors argue, bring added complexity to providing effective treatment for women and require responses that address substance use, interpersonal abuse, and PTSD symptoms in an integrated way rather than treating each issue in silo.

\section{Culture-informed approaches}

There is a dearth of evidence that considers specific culture-sensitive good practice when working with those from minority ethnic groups facing multiple disadvantage. There is a developing literature on engaging BAME communities in primary care services but not necessarily focused on those with multiple needs (see Brach \& Fraserirector, 2000; Salway, 2014). This is a significant gap because - as outlined in Chapter Three - social and cultural norms associated with an individual's ethnic identity impacts on their support journey. Cultural competence is therefore important in developing a trauma-informed approach to care (Hopper et al., 2009).

The existing evidence on culture-informed approaches for those facing multiple needs suggests a number of good practice recommendations. These range from practical suggestions around displaying information in a range of languages and ensuring interpreters are available, to taking a holistic approach and attending to the different and changing needs of the individuals they are working with, to being aware of the ways that distress might be presented differently $(\mathrm{EACH}, 2014$; Wilton \& Williams, 2019). It is also stated that services for people with multiple needs should also accommodate other factors that complicate beneficiaries' lives, making them physically and socially accessible to all, providing a range of communication methods, and making information easy to read and understand (Lamb et al., 2019a). Evidence states that the availability of good information, support and advocacy or a 'brokerage role' is particularly important for BAME communities, among whom access to and take up of services is lower proportionally than among the majority white population (Matharoo and Davis, 2007).

In Fish and Fakoussa (2018)'s study, the importance of mental health and well-being services that are accessible to females from cultures in which women are not permitted to go out on their own or enter vehicles and rooms alone with a male was discuss ed. Services and professionals who are sensitive to the additional issues faced by females from certain cultures were spoken of as vital. Linked with the perceived current lack of cultural and religious understanding within existing services, contributors felt that service users should and could have more input and leadership within developing services for their own needs.

Overall, these processes require services to commit to a 'culture of thoughtfulness' and communication in which they consider the different needs and experiences of those they are working with. Studies have shown that services need to make concerted efforts to meaningfully understand complexity of need by listening to the people they support and responding to them as individuals; where services have taken a shortcut and made assumptions about needs based on generic labels this was picked up by people and experienced as 'silencing' and 'uncaring' (Wilton \& Williams, 2019).

\subsection{Other Key Factors}

Adopting gender or culture-informed approaches on their own would be counterproductive if other factors that make up a trauma-informed care response are ignored. While the common principles of trauma-informed care are explored briefly at the beginning of this chapter, this section focuses on three factors that were identified as key in the literature: safe environments; staff training support and supervision; and empowering relationships. It is important to note that some of these ways of working are not gender-specific or culture-informed but reflect generally accepted principles of 
good trauma-informed care (Sweeney et al., 2016). They also chime with recommendations and guidelines around effective care for people with co-occurring mental health and drug use conditions which encourage strong therapeutic alliance, care that reflects the views, motivations and needs of the person and therapeutic optimism (Public Health England, 2017b).

\section{Safe environments}

The aim of PIEs is to create a network of relationships that enable people to find the sense of environmental and emotional safety [...] that has been missing for them for so long (perhaps even forever) and which in turn enables them to pursue their own recovery journey (Cockersell, 2011: 47).

The concept of the 'Psychologically Informed Environment' (PIE) was originally developed by Robin Johnson and Rex Haigh, as part of the Royal College of Psychiatrists' "enabling environment working group" which was set up in 2007-2008 and ran for 3 years. Johnson and Haigh (2010, p. 31) define the concept of a PIE as "the outcome of an attempt to identify, adapt and consciously use those features of the managed environment in such a way as to allow the resources and the day-to-day functioning of the service to be focused on addressing the psychological needs and emotional issues thrown up by the residents". The PIE concept followed on from the enabling environment concept as a solution to the needs that people with mental health and emotional problems have. Clients needed to feel physically and emotionally safe during treatment if they were to engage in trauma-informed treatment and to benefit from it.

The evidence on PIEs, while practice-based rather than from academic studies, suggests that PIEs are effective for people facing multiple disadvantage and with histories of compound trauma as well as the staff working with them (Cockersell, 2016). Data from St Mungo's PIEs shows that PIE residents were two and a half times less likely than non-PIE residents of a similar profile to be evicted; they were also 20 per cent more likely to have a positive move-on outcome (Cockersell, 2016). Cockersell (2016) also cites a Thames Reach and South London and Maudsley (SLaM) NHS Foundation Trust PIE partnership for homelesspeople in Lambeth, which found a 315 per cent increase in engagement with primary care and a 38 per cent increase in engagement with addiction services for PIE clients within six months of entering the PIE; within 18 months, 62 per cent of drug users and 75 per cent of drinkers were engaged with treatment.

Several academic, government, private and third sector initiatives were launched to improve homelessness services between 2008 and 2015 as part of a growing awareness that people experiencing homelessness present with various psychological and emotional needs and the realisation that services were often ill equipped to respond. This deviates from a traditional behavioural approach which uses a regime of warnings with eviction as the 'ultimate deterrent' (Cockersell, 2011). Psychological perspectives offer a different way of working with people.

'Housing First' re-houses people who have an entrenched history of sleeping rough into independent living from the outset of their engagement with a support provider and delivers 'floating support' in situ. This contrasts to more established 'staircase-toresettlement' models, which insist on 'treatment first' to people in temporary accommodation to ensure their 'readiness' for independent living. There is also a growing endorsement of Housing First as a gender-sensitive approach to housing that takes account of the diverse and complex situations of women. Evidence points to this approach working successfully with women who have experienced long-term homelessness (Busch-Geertsema, 2013; Pleace \& Bretherton, 2013). Housing First approaches recognise that women require targeted support in housing as well as 
ongoing and carefully managed interventions designed to support and maintain the transition to independent living (Mayock et al., 2015).

Emotional safety can only be fostered when physical safety is provided. For women who have experienced violence and abuse, the male-dominated nature of many day centres and mixed gender substance treatment services can make them threatening and frightening. Women-only spaces have been found to facilitate safety on both an emotional and physical level (DHSC, 2019; Holly, 2017). Tomkins and Neal (2018) recommend investing in suitable premises and facilities that enable women to feel comfortable and secure and to establish daily routines and structures that promote client saf ety and stability. There was a lack of evidence specifically relating to fostering safety in mixed gender spaces.

\section{Staff training, support and supervision}

There is a relatively short history of trauma-informed working in the UK when compared to the USA for example whose National Center for Trauma-Informed Care was established in 2005. Tomkins and Neal (2018) found alack of routine staff training in trauma-informed working in the UK and suggest that there is a need for financial resources to pay, train, support, and retain staff - especially where care is provided 24 hours a day. Moreover, training should ideally be provided to staff before they start working within a trauma-informed service. St. Mungo's has developed a range of training for staff at their PIE pilots (Cockersell, 2011). This includes client co-produced training on topics from attachment theory to motivational interviewing; management training so that managers can better support staff creativity; and the establishment of reflective practice groups. The essence of their approach is personalised, recoveryoriented, and reflective practice; staff are encouraged to ask themselves, 'did what I did with/said to this/these client(s) progress their recovery journey or create an obstacle in it?' If staff feel more empowered and confident in supporting people facing multiple disadvantage, this can lead to a reduction in the number of people being turned away from services on grounds of behaviour (DHSC, 2019).

The evidence also highlights psychologically informed planned environments (PIPES), specifically designed, contained environments where staff members have additional training to develop an increased psychological understanding of their work (Department of Health \& Ministry of Justice, 2012). This understanding enables them to create an enhanced safe and supportive environment, which can facilitate the development of those who live there. It is stressed that staff who work on PIPEs should be carefully selected and appropriately trained and provided with support and clinical supervision. Through training and support, staff will begin to develop an increased understanding of ordinary, everyday behaviour as well as more complex behaviours within a forensic setting.

It has been shown that staff working with groups with multiple needs are frequently exposed to traumatic material with consequences for their own mental health and challenges for managing personal distress and avoiding 'burn out'. A recent study highlights the elevated levels of stress and depression among frontline workers in homelessness services and calls for prioritising peer-led supervision, as well as earmarked funds that go directly to supporting staff mental health and wellbeing (Lemieux-Cumberlege and Taylor, 2019). Trauma-informed staff need to know how to maintain their personal and professional boundaries in order to protect their own wellbeing and be consistent in their approach towards people they support.

\section{Empowering relationships}

Good trauma-informed therapeutic relationships with individuals involves not judging, never giving up on them, treating them differently from how they were accustomed to 
being treated, and identifying what motivates them, and working collaboratively to produce treatment care plans tailored to their needs. A person-centred and individualised approach recognises that pathways of recovery and progress are different for everyone, and relapses and setback are inevitable. Services work well when they accommodate this reality rather than punish it by excluding people or closing cases (Lamb et al., 2019b). When women's traumatic responses and behaviours are explained and normalised by practitioners, evidence suggests that this makes a significant difference to their recovery and feelings of safety. It is therefore important to staff to understand how trauma manifests in behaviour, in addition to how to respond appropriately (MCDMC, 2018). Clients' previous experiences of traumaand their lack of familiarity in sharing their experiences may undermine their ability to trust people, which may affect relationships with staff. Some clients may not want, or feel able, to consider their past experiences of trauma (Tomkins and Neal, 2018). Appreciating the fact that women may not feel safe or ready to disclose traumatic personal information, questions about the potential issues affecting clients should be asked at different points in time and not just at an initial assessment (Williamson et al., 2013). Cultural and gender competence are well-established good practice principles yet practitioners do not always have insight into, identify or appreciate the effects of the power dynamics within which they work and the culture that exists to fix or rescue people in paternalistic and disempowering ways (Sweeney et al., 2018).

Some studies emphasise the importance of workers having 'lived experience' or direct personal experience of recovery as well as prior experience of working with the client group (NCDSV, 2019; NCDMC, 2018; Tomkins and Neal, 2018). For BAMER women, specialists in BAMER women's experiences are important so women can speak in their own language and be supported by women who understood what they have been through (NCDSV, 2019). Being able to choose the gender of practitioner and receive support in a women-only space has been cited as important for the recovery of women who have experienced abuse or violence (DHSC, 2018).

Prioritising client safety, particularly at the start of their treatment is crucial. Sharpen (2018) draws attention to the importance of body language in service encounters. She suggests that women who have experienced trauma are often hyper-vigilant and will pay close attention to body language, eye contact and active listening. Taking a holistic 'whole woman approach' - and not simply thinking in terms of need - is applied by WomenCentre when working with women and families facing complex issues (Duffy and Hyde, 2014).

The quality of relationships emerged as one of the most valued aspects of support by women in a review of literature by Holly (2017). This may also be the case for men too. Non-judgemental attitudes by staff were identified by both people accessing services and practitioners as being important for building trust and successful relationships. Reliability and trustworthiness were vital to women in Sharpen's (2018) study. There was a feeling from respondents that if you can't trust someone with the little things (i.e. ringing at an agreed time), there was no point trusting them with the real issues. Archard and Murphy's (2015) study provides an account of how mental health support work is experienced by homeless service users when it is informed by a person-centred, non-directive approach and implemented by trainee health and social care professionals under the auspices of a specialized psychological trauma service. Service users appreciated the face-to-face time support workers could, with relatively modest caseloads, invest in working alongside them. This was contrasted with the amount of time shelter staff could commit, who, whilst cordial and warm, were recognised as juggling the demands of several residents and administrative duties. 


\subsection{The Challenges of Implementing a Trauma-informed Approach}

Some studies have explored the barriers for services in delivering a trauma-informed approach to support. This may be due to funding and commissioning conditions. Many of the services visited in Bear et al.'s (2019) study faced challenges to delivering holistic support because of what they described as precarious or fragile funding conditions, of ten through a number of different funding streams. Most of their funding was relatively short term so these organ isations felt they were "constantly battling to survive". Other studies add weight to the argument that support services are increasingly compromised in responding to complex needs as a result of financial cuts to social welfare (Scullion et al., 2014). Reports also note that many public sector providers are commissioned through block contracts, with success largely based on volume of service delivery and an ability to meet targets rather than outcomes or the quality of a service (Bear et al., 2019). The authors noted how this focus is counter to the holistic model of support that practitioners argue is integral to working in a traumainformed way. In order to fully implement trauma-informed approaches it was stressed that the culture of commissioning needs to change; moves towards commissioning through a 'trauma lens' and joint commissioning models were suggested as potential solutions.

There are organisational barriers to implementing trauma-informed approaches, in that organisations may find it challenging to make the sort of cultural shift required in order to start delivering trauma-informed services (Bear et al., 2019). This tends to be the case for public sector organisations and some voluntary and community sector organisations more so than women's centres, which are generally more wellestablished in delivering trauma-informed approaches. The move to full "traumainformedness" involves all aspects of a service undergoing a process of change, from the senior leadership, staff training and the physical environment, to considering how all aspects of a service operate. Buy-in from all staff is integral. This buy-in could be achieved through informal education programmes on the benefits of trauma-informed working; the development of local toolkits; and service 'champions' who act as influencers (Bear et al., 2019).

An additional significant challenge includes the difficulty of bringing about a traumainformed approach in an inherently traumatising environment, such as a prison. Women who had participated in prison-based trauma programmes stressed that interventions would only work if the whole of the prison was trauma-informed otherwise the benefits of the programme would become undone (Bear et al., 2019). Positive impacts were also contingent on the continu ation of support after release from prison. 


\section{Good Practice Examples}

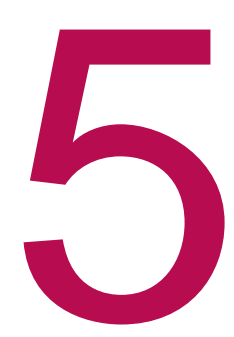

This chapter presents several good practice examples, from the literature, of effective trauma-informed care practices in services working with people facing multiple disadvantage.

1. Mapping the Maze model (Holly, 2017) is a framework for good practice for delivering interventions/services for wo men experiencing multiple disadvantage, to inform and guide the commissioning and delivery of services that meet their specific needs in a given geographical area. The Mapping the Maze model has four broad components: organisational ethos, s afe and enabling environment, approach to working and organisational practice:

A. Organisational ethos: commitment to delivering gender-responsive services and interventions.

This means:

- having specialist knowledge of women's lives and experiences

- recognising multiple disadvantage, including diversity issues

- und erstanding inter-related needs requiring individual holistic care

- recognising impact of trauma, particularly in terms of violence and victimisation

- accepting women - viewing behaviour as adaptation and resilience rather than symptoms and pathology

B. Safe and enabling environment: provision of support in places where women feel safe and welcome.

This means:

- women-only space

- physically safe, particularly when women may be affected by violence and abuse

- prioritising emotional safety that minimises the risk of re-traumatisation

- an environment that promotes dignity, self-respect and wellbeing

C. Approach to working: how interventions are delivered is as critical as what support is facilitated.

This means:

- safety, respect and acceptance are paramount

- trust is a key priority, built through consistent relationships

- $\quad$ working with the individual, including being culturally competent

- build on strengths and ways of coping

- enables choice and control, which in turn builds self-efficacy

- collaboration - building a plan with a service user not for, and working with other agencies

- offering time and flexibility

D. Organisational practice: structures are in place to enable gender-responsive interventions.

This means:

- recognising challenges of working with wo men experiencing multiple disadvantage

- providing sufficient staff support - informal and line management/clinical supervision

- continued staff development

- engaging with partners to develop integrated multi-agency responses

- challenging and working to eliminate causes of women's multiple disadvantage

- being aware of the need to develop cultural competence and address issues relating to intersectionality 
2. Homeless Health Peer Advocacy (HHPA). Groundswell founded HHPA to improve people's conf idence in using health services and increase their ability to access healthcare independently. At the core of their service are peer advocates who have experience of homelessness themselves and provide one-to-one support to attend health care appointments. The support of a trusted advocate, with similar experience, to overcome some of these challenges, is intended to significantly improve the health and wellbeing of people affected by homelessness. Groundswell is currently working with a team of academic partners to conduct research exploring the impact of HHPA. In 2016 Groundswell extended HHPA to support homeless people in tackling complex mental health needs. The scheme was led by volunteers with experience of homelessness who gained mental health peer support skills, training and clinical supervision to deliver with Ground swell 100 Health and Mental Health promotion sessions in hostels and day centres that reached 600 clients, 343 one-to-one engagements, and support for 66 appointments relating to mental health.

3. In 2007, the charity, the Revolving Doors Agency developed the Revolving Doors National Development Programme (NDP) (in Anderson, 2011) to specifically overcome the challenges of working with a hard-to-engage group. Their work was focused in the West Midlands with women using Anawim, a women's centre based in Birmingham that provides a holistic service to women across the city. The NDP worked at three levels: identification of needs, demonstration of solutions, and shaping policy and commissioning. Staff at Anawim observed that many of the women appeared to have some form of mental health need and/or had had previous contact with mental health services. However, current contact with these services was rare, and where occurring did not always appear to be meeting needs. Although Anawim has a counselling service (not open to people with severe and enduring mental health problems), prior to the pilot there was no in-house support from primary care or mental health services and Anawim staff lacked knowledge or conf idence to be able to help these women access the mental health support they needed. A steering group was convened consisting of representatives from Anawim, Birmingham and Solihull Mental Health Foundation Trust, Revolving Doors Agency, NHS West Midlands, the Pan Birming ham Mental Health Commissioning Team and the National Offender Management Service West Midlands. A Mental Health Nurse from the dual diagnosis field was seconded one day a week to Anawim. Her primary role was to assess the women's mental health needs and help them to access appropriate support. Anawim staff referred wo men to the MHN and women could selfref er or approach the MHN informally around the centre. 


4. St Basils Psychologically Informed Environments. A consistent psychological approach is
taken across a range of services including their bespoke Mental Skills Training for Life
Parenting Young
aspects of three main psychological approaches: (1) cognitive behaviour therapy (CBT), (2)
dialectical behaviour therapy (DBT), and (3) cognitive analytic therapy (CAT). This framework is
made explicit to staff in their foundation training and shapes a shared language and set of
expectations for staff to use in their day-to-day work.
Taking a holistic approach, staff create a physical and social environment that is affirming, caring,
empowering, and validating. Collaboration is also crucial, with every individual supported to
determine their own personal goals and achievements. Furthermore, young people are regularly
involved in making decisions about the environment in which they live, and this input is used to
guide the changes that are made.
St Basils provide their staff with ongoing training and support via formal courses, reflective
practice, and access to the in-house psychologist. One of the lessons learned in its evaluation was
that the quality of relationships matter. To break through any past history of adversity and
abandonment, and to develop genuine trust and rapport, there is a need for consistent and stable
contact between staff and young people. This type of relationship must be nurtured with time and
attention, which has practical implications for both staff workload and the length of time young
people stay at St Basils (Cumming et al., 2017).

5. 1625 Independent People (1625ip) is a housing and youth support organisation working with
homeless and insecurely housed young people aged from 16 to 25$)$ in Bristol and the surrounding
area (Woodcock and Gill, 2014). The majority of the young people enter their services following
homelessness, when leaving local authority care and custody and through involvement in the
criminal justice system. Their intention was to develop and model a PIE framed by a core
understanding that the "safe passage" of young people through the service is underpinned by an
experience of being "held" and emotionally "contained". Project workers while not therapists have a
basic understanding of how disrupted attachments can shape relational behaviour. Arising from
this, relationships with young people are considered the principal tool for change. Specialist
support is provided alongside young people's "key worker" and support lasts typically for a period of
three months to two years. In addition to the more generic support and sensitivity, project workers
use a range of different therapeutic work informed by thinking from different therapeutic modalities
including psychodynamic, systemic, humanistic, CBT and dialectical behaviour therapy (DBT).
Another very important strand of the work of the PIE is the development of peer support and
community mentoring. The model is supported by the development of physical environments
that support emotional well-being and an embrace of 1625 as a learning organisation
(Fieldhouse and Parmenter, 2017).

6. Lancashire Women's Centres (LWC) are based on the foundational principle that 'gender matters'. The centres provide services which recognise that women with complex needs need women workers and volunteers to work with at point of crisis, and need consideration of the experiences of trauma, abuse and exploitation that they may have experienced in their lives. LWC bring together a range of services under one roof, enabling the provision of evidence-based prog rammes in safe, accessible community settings, adopting a 'whole person' approach to address the impact of social, environmental, economic and psychological influences upon women's wellbeing. The core offer of services provided by LWC include therapeutic programmes, including IAPT provision, debt, money and housing advice, skills development support, and social inclusion activities. Their three programmes, 'Vision', 'Avert', and 'Achieve', were evaluated in 2016 (Codd et al., 2016). Vision was aimed at women within the criminal justice system and acted as an alternative sentencing option. Avert is a police custody-based liaison and diversion strategy that works with wo men to identify the triggers to offending and the underlying causes of offending. Achieve supports women in the criminal justice system and offers advice and guidance to help women move into sustainable employment. The participants of each programme all commented on the value of being able to talk openly without feeling as if they would be judged, either by staff or other participants. The fact that the programmes were run by and for women was highlighted as a specific strength of the programmes. The Women's Centre provides a wide range of services, and this holistic approach was recognised as highly important by the participants, who appreciated the combination of different forms of support. Evidence suggests that participation in the programmes led to reduced reoffending, as well as having a positive impact on depression and anxiety. 
7. Tomorrow's Women Glasgow (McKechnie, 2016) is a one-stop shop to meet the complex needs of high risk female offenders, established by Glasgow City Council, in partnership with Greater Glasg ow and Clyde Health Board, Glasgow Housing Association and the Scottish Prison Service. At the planning stage, all involved were aware of the high incidence of abuse and trauma that was a key feature in the development of women's difficulties. Referrals come from criminal justice, social work and prisons; the women have high levels of need, often homeless, with histories of poor contact with health and social work, experience difficulties with family contact and are burdened with substance misuse problems. Within Tomorrow's Women Glasgow the environment is key to providing the basics of a Trauma-Informed Approach; rooms are light, airy and decorated with art work prepared by the wo men, alongside photographs illustrating activities and achievements. Staff are always available to welcome women, safety being relational rather than physical. All women are allocated a key worker who completes a care plan with each woman, identifying and prioritising their need. Engaging the women with the service involves frequent phone calls, text messages, home visits and close liaison with other agencies. Tomorrow's Women Glasgow prioritises supervision for staff, provided four weekly by the team lead. In addition, they have established weekly complex case discussions and regular training sessions so that staff, even if they are not directly 'treating' symptoms of Complex PTSD, develop a trauma-informed understanding of the women's presenting problems. Once women have established trust with the service, they can be referred for assessment and treatment of their mental health problems, be it Complex PTSD, clinical depression, chronic anxiety or personality difficulties. This stage involves more direct engagement with clinical psychology, where traumatic memories are explored in detail and the woman helped to change her views of the abuse.

8. The Nelson Trust (DHSC, 2019). The Nelson Trust's Women's Services based in Gloucestershire, Swindon, Wiltshire, Bristol and Somerset provide holistic, trauma-informed and gender-responsive services. Every woman is offered a detailed individual assessment of their needs across nine 'pathways'. These include accommodation, physical and mental health, drugs and alcohol, finance and benefits, family and relationships, domestic abuse, sex work, education and training, attitudes, and thinking and behaviour. They are each provided with their own key worker to help in developing their own needs-specific support plans, in addition to individual and group sessions and access to specialist services across the county. They also have access to a timetable of activities, including accredited educational courses and workshops, and an onsite crèche, showers, washing machine, garden and cafe. The Nelson Trust has a number of bespoke projects addressing the needs of women and girls affected by sexual exploitation and abuse. 


\section{Key Evidence Gaps}

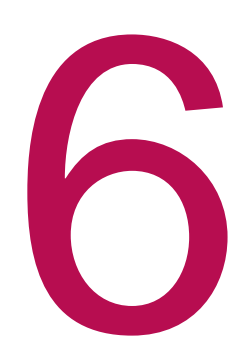

This last chapter brings together the key evidence gaps that the review has highlighted.

\subsection{Service Access}

There is now a sizeable array of sources on multiple needs and exclusions, with a growing recognition that there is a significant overlap between groups subject to multiple and extreme forms of disadvantage. However, with exceptions, studies which provide robust evidence on the profile, nature, and extent of severe and multiple disadvantage are scarce. Much of the literature is in the form of small-scale studies by voluntary organisations focused on their particular client group. There is a clear need, therefore, for larger-scale, national studies which provide a more detailed picture of relevant overlaps to further evidence the case that people facing multiple disadvantage require effective and co-ordinated services. Evaluation of the national Fulfilling Lives programme and the MEAM programme are building a stronger evidence base.

While there is a relatively large body of evidence on systemic barriers to service access for those facing multiple disadvantage, it tends to be fragmented into different groups, some of which receive more focus than others in the literature. In a clear break from older studies, which have been criticised for neglecting issues of gender by subsuming their usually small number of female participants into a larger male sample, there is a plethora of recent research focusing solely on women with multiple needs and their access to services. This literature has a tendency to treat women with multiple needs as one group; there is much less evidence on barriers for BAME or disabled women facing multiple disadvantage, for instance. Further, there is more work that needs to be done on specific barriers to service access and productive support encounters for particular groups facing multiple disadvantage, most obviously black and ethnic minorities, LBGT+ people, and people with disabilities.

It is clear from this albeit limited evidence base that there are gaps in understanding in some services around meeting specific needs and accommodating different experiences. The consequences of not doing so are severe. The net effect of this can reinforce an 'inverse care law'; i.e. those with the greatest needs are at greatest risk of getting the least services (Rosengard et al., 2007). Evidence hints that flexible and personalised support as being the solution but is not robust. There needs to be a deeper understanding of what people actually want, based on studies grounded in the perspectives of people facing multiple disadvantage. 


\subsection{Social and Cultural Norms and Support Journeys}

While for a long time definitions of multiple disadvantage have been inadvertently framed by the experiences of men, there is a growing body of literature focussed on the experiences of women. Within this, some attention is paid to the experiences of BAME women but this is minimal. Reflecting the smaller number working with services, BAME women commonly comprise only a small cohort of research samples. There is a need for more in-depth research to understand multiple disadvantage from the perspective of people from different BAME communities.

The particular experiences of BAME men are largely absent from the evidence base on multiple disadvantage. It is likely that in many ways their support pathways will be significantly different to those of white British men, not least because of socioeconomic disadvantage, racism and historical trauma. Research is therefore also needed which focuses on the specific and particular experiences of BAME men.

\subsection{Trauma-informed Policy and Practice Responses}

While there is a bourgeoning evidence base on trauma-informed interventions for those facing multiple disadvantage, it remains under-developed in some respects. Firstly, it tends towards offering descriptive accounts of what constitutes traumainformed practice rather than critically evaluating or theorising such approaches and their effectiveness. Dobson (2019: 20) poses the question, for example, 'what do models that embrace complexity delimit and restrict as well as enable for practitioners in their day-to-day thinking about social problems, human experiences and support practice?' Secondly, the evidence is not developed enough to answer whether traumainformed responses used with people facing multiple disadvantage take into account the variations and complexities of experience between different groups with different support journeys and needs. While the literature on gender-specific responses is now more established, there is less that considers race and how services might become culture-informed.

The evidence highlights the importance of services meaningfully engaging with people they support to understand their needs and preferences around service design and delivery, and what they feel a trauma-informed approach should look like. However, evidence which asks these questions of different groups of people is currently lacking. 


\section{References}

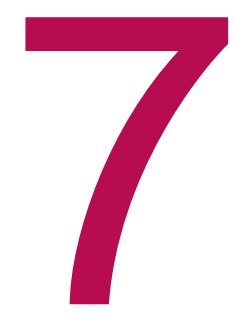

Agenda (2016) Women's Needs in Mental Health Services: A Response to a Freedom of Information Request. London: Agenda.

Agenda (2018) Women in Crisis: How Women and Girls are being failed by the Mental Health Act. London: Agenda.

Aldridge RW, Menezes D, Lewer D et al. (2019) Causes of death among homeless people: a population-based cross-sectional study of linked hospitalisation and mortality data in England. Wellcome Open Res, (4), pp. 49.

(https://doi.org/10.12688/wellcomeopenres.15151.1)

Anderson, S. (2011) A way through the woods: opening pathways to mental health care for women with multiple needs. Advances in Dual Diagnosis, 4 (2), pp.63-74.

Archard, P.J. and Murphy, D. (2015) A practice research study concerning homeless service user involvement with a programme of social support work delivered in a specialized psychological trauma service. Journal of Psychiatric and Mental Health Nursing, 22, pp. 360370.

Ava and Agenda (2017) The Core Components of a Gender Sensitive Service for Women Experiencing Multiple Disadvantage:A Review of the Literature. London: AVA and Agenda.

Ava and Agenda (2018) Hand in Hand: Survivors of Multiple Disadvantage discuss Service and Support. London: Ava \& Agenda.

Ava and Agenda (2019) National Commission on Domestic and Sexual Violence and Multiple Disadvantage: Breaking Down the Barriers. London: Ava \& Agenda.

Bailey, K., Trevillion, K. and Gilchrist, G. (2019a) "We have to put the fire out first before we start rebuilding the house": practitioners' experiences of supporting women with histories of substance use, interpersonal abuse and symptoms of post-traumatic stress disorder. Addiction Research \& Theory, DOI: 10.1080/16066359.2019.1644323

Bailey, K., Trevillion, K. and Gilchrist, G. (2019b) What works for whom and why: A narrative systematic review of interventions for reducing post-traumatic stress disorder and problematic substance use among women with experiences of interpersonal violence. Journal of Substance Abuse Treatment, 99, pp. 88-103.

Bashir, N., Ameyaw, N., Sahota, S, Bajwa, M and Dayson, C. (2019) Culture, connection and belonging: a study of addiction and recovery in Nottingham's BAME community. BACIN, Lankelly Chase and Sheffield Hallam University.

Bear, L., Durcan, G. and Southgate, J. (2019) A sense of safety: Trauma-informed approaches for women. London: Centre for Mental Health.

Bowpitt, G., De Motte, C., Ledgister, C., Spours, J., Walsh, R., Everitt, G., Kaur, K (2018) Multiple needs: meeting the challenge, Opportunity Nottingham year four (midway) report, 2014-2022. Nottingham: Nottingham Trent University. 
Bowpitt, G., Dwyer, P., Sundin, E. and Weinstein, M. (2011) Comparing Men's and Women's Experiences of Multiple Exclusion Homelessness. Social Policy \& Society, 10 (4), pp. 537 546

Brach, C. and Fraserirector, I. (2000) Can cultural competency reduce racial and ethnic health disparities? A review and conceptual model. Medical Care Research and Review, 57 (4), pp. 181-217.

Bramley, G., Fitzpatrick, S., Edwards, J., Ford, D., Johnsen, S., Sosenko, F. and Watkins, D. (2015) Hard Edges: Mapping severe and multiple disadvantage. London: Lankelly Chase Foundation.

Breedvelt, J. (2016) Psychologically Informed Environments: A Literature Review. London: Mental Health Foundation.

Busch-Geertsema, V. (2013) Housing First Europe: Final Report. Available at http://www.habitat.hu/files/FinalReportHousingFirstEurope.pdf

Byrne, B., Alexander, C., Khan, O., Nazroo, J., and Shankley, W (Eds) (2020) Ethnicity, Race and Inequality in the UK: State of the Nation. Bristol: Policy Press.

Cameron, A., Abrahams, H., Morgan, K., Williamson, E. and Henry, L. (2016) From Pillar to Post: Homeless women's experiences of social care. Health and Social Care in the Community, 24 (3), pp. 345-352.

Cattell, J., Mackie, A., Gibson, K., Hitchins, T., Parry, W., Porsch, L., and Savage, J. (2011) Simple but effective: Local solutions for adults facing multiple deprivation. London:

Department for Communities and Local Government.

CFE Research and The University of Sheffield (2020) Improving access to mental health support for people experiencing multiple disadvantage. London: The National Lottery Community Fund.

Changing Lives (2018) Too Complex for "Complex Needs"? Learning from work with victims of domestic abuse, who also have multiple and complex needs. London: Changing Lives.

Clinks (2013) Run Ragged: The current experience of projects providing community based female offender support services. London: Clinks.

Cockersell, P. (2011) More for less? Using PIEs and recovery to improve efficiency in supported housing. Housing, Care and Support, 14 (2), pp. 45-50.

Cockersell, P. (2016) PIEs five years on. Housing, Care and Support, 20 (4), pp. 221-230.

Codd, H., Doherty, J., Doherty, P., Robertson, L. and Elliott, A. (2016) An Evaluation of the 'Vision', 'Avert', and 'Achieve' Interventions. Preston: University of Central Lancashire.

Covington, S.S. (2016) Becoming Trauma Informed Tool Kit for Women's Community Service Providers. London: Centre for Crime and Justice Studies.

Crisis (2018) Everybody In: How to End Homelessness in Great Britain. London: Crisis.

Cumming, J., Skeate, A. and Anderson, G. (2017) St Basils Psychologically Informed Environments - Meeting the emotional and psychological needs of young homeless people. London: Housing Learning \& Improvement Network.

Department of Health and Ministry of Justice (2012) A Guide to Psychologically Informed Planned Environments (PIPES). London: Department of Health.

DeVerteuil, G. (2003) Homeless mobility, institutional settings, and the new poverty management. Environment and Planning A, 35, pp. 361-379.

Department Health and Social Care (2019) Breaking Down the Barriers: Findings from the Commission on women facing domestic and sexual violence and multiple disadvantage. London. 
DHSC (2018) The Women's Mental Health Taskforce final report. Available at:

https://assets.publishing.service.gov.uk/government/uploads/system/uploads/attachment da ta/file/765821/The Womens Mental Health Taskforce - final report1.pdf

Dobson, R. (2019) Complex needs in homelessness practice: a review of 'new markets of vulnerability'. Housing Studies, DOI: 10.1080/02673037.2018.1556784.

Duffy, S. and Hyde, C. (2014) Women at the Centre: Innovation in Community. Sheffield:

The Centre for Welfare Reform.

Duncan, M. and Corner, J. (2012) Severe and Multiple Disadvantage: A Review of Key

Texts. London: Lankelly Chase Foundation.

EACH (2014) Asian Women, Domestic Violence and Mental Health: A Toolkit for Health Professional. London: EACH.

Elliott, I. (2016) Poverty and Mental Health: A review to inform the Joseph Rowntree Foundation's Anti-Poverty Strategy. York: JRF.

Fish, M. and Fakoussa, O. (2018) Towards culturally inclusive mental health: learning from focus groups with those with refugee and asylum seeker status in Plymouth. International Journal of Migration, Health and Social Care, 14 (4), pp. 361-376.

Fieldhouse, J and Parmenter, V (2017) A summary of UWE's evaluation of 1625 independent people's Cash Pointers Service. UWE.

Fitzpatrick, S., Johnsen, S. and White, M. (2011) Multiple exclusion homelessness in the UK: key patterns and intersections. Social Policy \& Society, 10 (4), pp. 501-512.

Gilburt, H. (2015) Mental Health under Pressure. The King's Fund. Available at:

https://www.kingsfund.org.uk/sites/default/files/field/field publication file/mental-healthunder-pressure-nov15 0.pdf

Gilburt, H. (2018) Funding and staffing of NHS mental health providers: still waiting for parity. Available at: https://www.kingsfund.org.uk/publications/funding-staffing-mental-healthproviders

Harris, M. and Fallot, R. (2001) Using trauma theory to design service systems. SanFrancisco: Jossey-Bass.

Holly, J. (2017) Mapping the Maze: Services for women experiencing multiple disadvantage in England and Wales. London: Agenda \& AVA.

Holly, J. and Lousley, G. (2014) The challenge of change - improving services for women involved in prostitution and substance use. Advances in Dual Diagnosis, 7 (2), pp. 80-89.

Homeless Link (2019) Youth Homelessness: Scoping report July 2019. London: Homeless Link.

Homeless Link (2018a) The Future Hostel: The Role of Hostels in Helping to End Homelessness. London: Homeless Link.

Homeless Link (2018b) Promising practice from the frontline: Exploring gendered approaches to supporting women experiencing homelessness and multiple disadvantage. London: Homeless Link and Women's Resource Centre.

Hopper, E.K., Bassuk, E.L. and Olivet, J. (2009) Shelter from the Storm: Trauma-Informed Care in Homelessness Services Settings. The Open Health Services and Policy Journal, 2, pp. 131-151.

Johnsen, S. and Tiexeira, L. (2010) Staircases, Elevators and Cycles of Change. London: Crisis.

Lamb, H., Moreton, R., Welford, J., Leonardi, S., O'Donnell, J. and Howe, P. (2019a)

Understanding Multiple Needs. London: The National Lottery Community Fund. 
Lamb, H., Moreton, R., Welford, J., Leonardi, S., O'Donnell, J. and Howe, P. (2019b) What makes a Difference. London: The National Lottery Community Fund.

Lamb, H., Moreton, R., Welford, J., Leonardi, S., O'Donnell, J. and Howe, P. (2019c) Why we need to invest in multiple needs. London: The National Lottery Community Fund.

Lemieux-Cumberlege, A. and Taylor, E.P. (2019) An exploratory study on the factors affecting the mental health and well-being of frontline workers in homeless services. Health \& Social Care in the Community, 27, pp. e367-e378.

Lucas, S., Archard, P.J., Tangen, J. and Murphy, D. (2018) Arrangements for adult service users who are homeless in English mental health trusts. Mental Health Review Journal, 23 (1), pp. 64-71.

MacLellan, J., Surey, J., Abubakar, I., and Stagg, H. (2015) Peer Support Workers in Health: A Qualitative Metasynthesis of their Experiences. PLoS ONE, 10 (10), e0141122. doi:10.1371/journal.pone.0141122

Magwood, O., Leki, V.Y., Kpade, V., Saad, A., Alkhateeb, Q., Gebremeskel, A., Rehman, A., Hannigan, T., Pinto, N., Sun, A.H., Kendall, C., Kozloff, N., Tweed, E.J., Ponka, D. and Pottie, K. (2019) Common trust and personal safety issues: A systematic review on the acceptability of health and social interventions for persons with lived experience of homelessness. PLoS ONE, 14 (12), pp. e0226306.

https://doi.org/10.1371/journal.pone.0226306

Matharoo, D. and Davis, S. (2007) Equality, Diversity and Housing: Future Prospects. Journal of Integrated Care, 15 (5), pp. 34-38.

Mayock, P., Sheridan, S. and Parker, S. (2015) 'It's just like we're going around in circles and going back to the same thing ...': The Dynamics of Women's Unresolved Homelessness. Housing Studies, 30 (6), pp. 877-900.

McClenaghan, M. (2019) Homelessness Kills: Study finds Third of Homeless People Die from Treatable Conditions [online]. Available at:

https://www.thebureauinvestigates.com/stories/2019-03-11/homelessness-kills

McKechnie, A. (2016) Tomorrow's Women Glasgow. Scottish Justice Matters, 4 (2), pp. 23 24.

McManus, S., Scott, S. and Sosenko, F. (2016) Joining the Dots: the combined burden of violence, abuse, poverty in the lives of women. London: Agenda

McNeish, D. and Scott, S. (2014) Women and Girls at Risk: Evidence across the Life Course. North Dalton: DMSS Research.

Mental Health Foundation (2016) Fundamental Facts about Mental Health2016. London: Mental Health Foundation.

Mental Health Foundation (2017) While Your Back was Turned: How mental health policymakers stopped paying attention to the specific needs of women and girls. London, Glasgow and Cardiff: Mental Health Foundation.

Ministry of Justice (2017) The Lammy Review: An independent review into the treatmentof, and outcomes for, Black, Asian and Minority Ethnic individuals in the Criminal Justice System. London: Ministry of Justice.

Muslim Women's Network UK (MWNUK) (2018) Written Evidence: National Commission into women facing domestic and/or sexual violence and multiple disadvantage. Birmingham.

ODPM (2005) Causes of Homelessness Amongst Ethnic Minority Populations. London.

Oram, S., Capron, L. and Trevillion, K. (2016) Promoting Recovery in Mental Health:

Evaluation Report. London: Kings College London.

Pleace, N. and Bretherton, J. (2013) Camden Housing First: A 'Housing First' Experiment in London. York: University of York. 
Public Health England (PHE) (2017a) Better care for people with co-occurring mental health and alcohol/drug use conditions: A guide for commissioners and service providers. London.

Public Health England (2017b) Mental health: population factor. In: Mental Health and Wellbeing: JSNA toolkit. London.

Reeve, K. (2018) Women and Homelessness: putting gender back on the agenda. People Place and Policy, 11 (3), pp. 165-174.

Reeve, K., Casey, R. and Goudie, R. (2006) Homeless Women: Still Being Failed yet Striving to Survive. London: CRISIS.

Reeve, K., Goudie, G. and Casey, R. (2007) Homeless Women: Homelessness Careers, Homelessness Landscapes. London: CRISIS.

Revolving Doors Agency (2015) Comprehensive Services for Complex Needs: A Summary of the Evidence. London: Revolving Doors Agency.

Robinson, M., Robertson, S., Steen, M., Raine, G. and Day, R. (2015) Doing and rethinking. Building resilience with men. Mental Health Review Journal, 20 (3), pp. 185-198.

Rose, D., Trevillion, K., Woodall, A., Morgan, C., Feder, G. and Howard, L. (2011) Barriers and facilitators of disclosures of domestic violence by mental health service users: qualitative study. The British Journal of Psychiatry, 198, pp. 189-194.

Rosengard, A., Laing, I., Ridley, J. and Hunter, S. (2007) A Literature Review on Multiple and Complex Needs. Edinburgh: Scottish Government.

Salway, S. (2014) Guest editorial: Ethnic diversity: opportunities and challenges for nursing research. Journal of Research in Nursing, 19 (6), pp. 456-459.

Scott, S. and McManus, S. (2016) Hidden hurt: Violence, abuse and disadvantage in the lives of women [Online]. Agenda. Available at: https://weareagenda.org/wpcontent/uploads/2015/11/Hidden-Hurt-full-report1.pdf

Scullion, L., Somerville, P., Brown, P. and Morris, G. (2014) Changing homelessness services: Revanchism, "professionalisation" and resistance. Health \& Social Care in the Community, 23 (4), pp. 419-427.

Sharpen, J. (2018) Jumping through hoops: How are coordinated responses to multiple disadvantage meeting the needs of women? London: AVA, MEAM, Agenda and St Mungo's.

Sheikh, S. and Teeman, D. (2018) A Rapid Evidence Assessment of What Works in Homelessness Services. London: Social Care Institute for Excellence.

Social Exclusion Taskforce (2006) Reaching out: an action plan on social exclusion. London: Cabinet Office.

Sosenko, F., Bramley, G., \& Johnsen, S. (2020) Gender Matters: Gendered patterns of severe and multiple disadvantage in England. Lankelly Chase Foundation.

https://lankellychase.org.uk/wp-content/uploads/2020/02/Gender-Matters-full-report-Feb2020.pdf

St Mungo's. (2014) Rebuilding Shattered Lives. London: St Mungo's.

Sweeney, A., Clement, S., Filson, B. and Kennedy, A. (2016) Trauma-informed mental healthcare in the UK: what is it and how can we further its development? Mental Health Review Journal, 21, pp. 174-192.

Sweeney, A., Filson, B., Kennedy, A., Collinson, L. and Gillard, S. (2018) A paradigm shift: relationships in trauma-informed mental health services. BJPsych Advances, 24, pp. 319333.

Sweeney, A., Perôt, C., Callard, F., Adenden, V., Mantovani, N. and Goldsmith, L. (2019) Out of the silence: towards grassroots and trauma-informed support for people who have experienced sexual violence and abuse. Epidemiology and Psychiatric Sciences, 28, pp. 598-602. 
The Young Foundation (2016) Saving Lives, Saving Money: How Homeless Health Peer Advocacy Reduces Health Inequalities. London: The Young Foundation.

Tompkins, C. and Neale, J. (2018) Delivering trauma-informed treatment in a women-only residential rehabilitation service: Qualitative study. Drugs: Education, Prevention and Policy, 25 (1), pp. 47-55.

Vaswani, N. and Paul, S. (2019) 'It's Knowing the Right Things to Say and Do': Challenges and Opportunities for Trauma-informed Practice in the Prison Context. The Howard Journal, 58 (4), pp. 513-534.

Walsh, C. (2020) Psycho-Social Approach for Understanding and Addressing Male Youth Violence: The Interface between Traumatic Experiences and Masculinity. Journal of Aggression, Maltreatment \& Trauma, 29 (2), pp. 186-205.

Williamson, E., Cameron, A. M., Morgan, K. J. and Abrahams, H.A. (2013) The TARA Project: A longitudinal study of the service needs of homeless women: Research Findings: The TARA Project. London: NIHR SSCR.

Wilson, B. and Astley, P. (2016) Gatekeepers: Access to primary care for those with multiple needs. Stoke-on-Trent: VOICES, Healthwatch and Expert Citizens.

Wilton, J. and Williams, A. (2019) Engaging with Complexity: Providing effective traumainformed care for women. London: Centre for Mental Health.

Woodcock, J. and Gill, J. (2014) Implementing a psychologically informed environment in a service for homeless young people. Housing, Care and Support, 17 (1), pp. 48-57. 


\section{Sheffield Hallam University}

Understanding Models of Support for People Facing Multiple Disadvantage: A Literature Review

MCCARTHY, Lindsey <http://orcid.org/0000-0002-5114-4288>, PARR, Sadie <http://orcid.org/0000-0002-1538-4807>, GREEN, Stephen <http://orcid.org/0000-00027813-0564> and REEVE, Kesia <http://orcid.org/0000-0003-2877-887X>

Available from the Sheffield Hallam University Research Archive (SHURA) at:

http://shura.shu.ac.uk/27186/

\section{Copyright and re-use policy}

Please visit http://shura.shu.ac.uk/27186/ and http://shura.shu.ac.uk/information.html for further details about copyright and re-use permissions. 